\title{
IMAGINARIES IN SEPARABLY CLOSED VALUED FIELDS
}

\author{
MARTIN HILS, MOSHE KAMENSKY, AND SILVAIN RIDEAU
}

\begin{abstract}
We show that separably closed valued fields of finite imperfection degree (either with $\lambda$-functions or commuting Hasse derivations) eliminate imaginaries in the geometric language. We then use this classification of interpretable sets to study stably dominated types in those structures. We show that separably closed valued fields of finite imperfection degree are metastable and that the space of stably dominated types is strict pro-definable.
\end{abstract}

\section{INTRODUCTION}

Since the work of Ax-Kochen and Ershov on the model theory of Henselian valued fields of residue characteristic 0 , yielding, e.g., the approximative solution of Artin's conjecture via the famous transfer principle between $\mathbb{Q}_{p}$ and $\mathbb{F}_{p}((t))$ for large $p$, valued fields have been a constant source for deep model-theoretic applications. One may mention here Denef's rationality results for certain Poincaré series, and the foundations of motivic integration.

But it is only with the work of Haskell, Hrushovski and Macpherson [8, 9] on the model theory of algebraically closed valued fields (ACVF) that the methods of geometric model theory have been made available for the study of valued fields. Then, in their groundbreaking work ([14], see also [6]), Hrushovski and Loeser used these newly available tools to give a new account of the topological tameness properties of the Berkovich analytification $\mathbf{V}^{a n}$ of a quasi-projective algebraic variety V. The purpose of this paper is to study separably closed valued fields of finite imperfection degree from the point of view of geometric model theory, in the light of these results.

Our first goal is the classification of the interpretable sets, also called imaginary sorts. Recall that a set is interpretable if it is the quotient of a definable set by a definable equivalence relation. A theory is said to eliminate imaginaries if for every interpretable set $\mathbf{X}$ there is a definable bijection between $\mathbf{X}$ and a definable set, in other words, if the category of definable sets is closed under quotients. In valued fields, contrarily to what happens, e.g., in various contexts of algebraically

Date: September 29, 2017.

2010 Mathematics Subject Classification. Primary: 03C60; Secondary: 12J20, 03C45, 03C98, $03 \mathrm{C} 10$

Key words and phrases. Model Theory, Separably Closed Valued Fields, Classification of Imaginaries, Stable Domination.

The first author was partially supported by ANR through ValCoMo (ANR-13-BS01-0006) and by DFG through SFB 878 .

The second author was partially supported by ISF through grant no. $1382 / 15$, and by ERC through FP7/2007-2013 (ERC Grant agreement no. 291111). The latter grant also funded a visit of the first author to Jerusalem in spring 2015 during which part of this research was carried out.

The third author was partially supported by ANR through ValCoMo (ANR-13-BS01-0006). 
closed fields with operators, it is not the case, in general, that the interpretable sets can all be understood in terms of the definable subsets of Cartesian powers of the field. There are some truly new quotients that appear and, in the case of ACVF, these new quotients are all described by Haskell, Hrushovski and Macpherson [8] as certain quotients of linear algebraic groups by definable subgroups. They show that it is enough to add to the valued field sort, for any $n \geq 1$, the set of $\mathcal{O}$-lattices in $K^{n}$, which is given by $\mathbf{G L}_{n}(K) / \mathbf{G} \mathbf{L}_{n}(\mathcal{O})$, as well as the union of all $s / \mathfrak{m} s$, where $s$ is an $\mathcal{O}$-lattice in $K^{n}$. Here $\mathcal{O}$ denotes the valuation ring of $K$ and $\mathfrak{m}$ denotes the maximal ideal of $\mathcal{O}$. These sorts are called the geometric sorts and we will denote them by $\mathcal{G}$.

Since then, it has been shown that various theories of valued fields eliminate imaginaries down to the geometric sorts, namely the theory RCVF of real closed valued fields (by work of Mellor [18]), the theories of $p$-adic fields and of ultraproducts of $p$-adic fields (by work of Hrushovski, Martin and the third author [15]), and the theory $\operatorname{VDF}_{\mathcal{E} \mathcal{C}}$ of existentially closed valued differential fields $(K, v, \partial)$ of residue characteristic 0 satisfying $v(\partial(x)) \geq v(x)$ for all $x$ (by work of the third author [23]).

Let us now consider separably closed valued fields. Recall that if $K$ is a non perfect separably closed field of characteristic $p>0$, then $\left[K: K^{p}\right]=p^{e}$ for some $e \in \mathbb{N}^{*} \cup\{\infty\}$, and the elementary theory of $K$ is determined by $e$, the so-called imperfection degree or Ershov invariant of $K$. Hrushovski's model-theoretic proof of the relative Mordell-Lang Conjecture in positive characteristic [13] illustrates that the theory of separably closed non perfect fields - a stable theory - is a modeltheoretic framework which provides a very useful approach for the study of questions from (arithmetic) algebraic geometry in positive characteristic.

In the valued context, the completions of the theory SCVF of separably closed non-trivially valued fields are determined, as in the case without valuation, by the imperfection degree. One also has an explicit description of the definable sets, by work of Delon and, more recently, work of Hong [12].

Our first result is that, as in the case of ACVF, the geometric sorts are sufficient to describe all the interpretable sets in separably closed valued field of finite degree of imperfection:

Theorem A (Theorem 3.9). The theory $\mathrm{SCVF}_{p, e}$ of separably closed valued fields of finite degree of imperfection e, with the elements of a p-basis named by constants, eliminates imaginaries down to the geometric sorts.

We prove this rather directly, reducing first to semi-algebraic sets using $\lambda$ functions and then performing a topological reduction to the corresponding result in $\mathrm{ACVF}_{p, p}$. The crucial ingredient is Hong's density theorem from [11] whose proof we include for convenience.

However, it seems more appropriate for practical purposes to work in the (strict) reduct obtained when, instead of working over a $p$-basis, one adds a sequence of $e$ commuting Hasse derivations (see [30]) to the language of valued fields. The situation is much trickier in this context. It is no longer the case that a definable subset of a Cartesian power of the field is necessarily in definable bijection with a semi-algebraic set. In order to reduce questions to $\mathrm{ACVF}_{p, p}$, prolongations come into the picture. In Corollary 4.23, we prove that the analogue of Theorem A also holds in $\mathrm{SCVH}_{p, e}$, the theory of existentially closed valued fields with $e$ commuting Hasse derivations. 
Note that Theorem A follows formally from this second result, but we chose to present both proofs as the shorter topological proof seems interesting and instructive to us. The first proof consists in finding a canonical way of representing a semi-algebraic set definable in a separably closed valued field $K$ by the $K$-points of a set definable in $\bar{K} \models \mathrm{ACVF}_{p, p}$. The second proof, inspired by the work of the third author on the theory $\operatorname{VDF}_{\mathcal{E C}}([23])$, is much more local. We only achieve a correspondence between $K$ and $\bar{K}$ at the level of types.

The main technical result which allows us, in the second proof, to reduce questions about definable sets to questions about types is the following density result for definable types, with parameters from the geometric sorts:

Theorem B (Theorem 4.21). Let $K \models \mathrm{SCVH}_{p, e}$, and let $\mathbf{X} \subseteq \mathbf{V F}^{n}$ be a $K$ definable set. Let $A=\mathcal{G}\left(\operatorname{acl}^{\mathrm{eq}}(\ulcorner\mathbf{X}\urcorner)\right)$. Then, there exists an A-definable type $p$ such that $p(x) \vdash x \in \mathbf{X}$.

Here, $\ulcorner\mathbf{X}\urcorner$ denotes the canonical parameter of $\mathbf{X}$ in $K^{\mathrm{eq}}$. Recall that a type $p(x)$ over some structure $M$ is said to be definable if for every formula $\varphi(x ; y)$ there exists a formula $\theta(y)$ over $M$, usually denoted by $\mathrm{d}_{p} x \varphi(x ; y)$, such that for all $m \in M$ :

$$
\varphi(x ; m) \in p \text { if and only if } M \models \theta(m) .
$$

The proof of Theorem B follows the same strategy as in the case of $\mathrm{VDF}_{\mathcal{E C}}$ mentioned above. The main new technical point is a quantifier elimination result for dense pairs of valued fields satisfying certain conditions. We then show that the pair $(\bar{K}, K)$, for $K$ a model of $\mathrm{SCVH}_{p, e}$, satisfies these conditions.

It follows immediately from this density statement that $\mathrm{SCVH}_{p, e}$, considered in the geometric sorts $\mathcal{G}$, has weak elimination of imaginaries. That finite sets are coded in $\mathcal{G}$ can easily be transferred from the corresponding result in $\mathrm{ACVF}_{p, p}$. This approach also has the added benefit of giving us, as a by-product, the fact that any type over an algebraically closed set has an automorphism invariant global extension, which is an important technical result for what follows.

In order to be able to classify imaginaries in the language of valued fields alone (in the case of finite imperfection degree, or even in the case of infinite degree of imperfection), it seems that one would need new ideas. In these cases, the goal would be to give a classification relative to those imaginaries which are definable in the field without valuation.

The second part of this paper is devoted to studying metastability and stably dominated types, first introduced by Haskell, Hrushovski and Macpherson [8, 9] to prove elimination of imaginaries in ACVF down to the geometric sorts. A type is said to be stably dominated if its "generic" extensions are controlled by pure stable interpretable subsets of the structure. A typical example is the generic type of the valuation ring $\mathcal{O}$ which is controlled by the residue map. Haskell, Hrushovski and Macpherson show that in a model of ACVF, over the value group, there are "many" stably dominated types. One says that ACVF is metastable and this gives a formal meaning to the idea that a model of ACVF is controlled in a very strong sense by its value group and its residue field. Precise definitions can be found in Section 6 .

In [14], Hrushovski and Loeser use the machinery of geometric model theory in ACVF to construct a model-theoretic avatar $\widehat{\mathbf{V}}$, whose points are given by the stably dominated types concentrating on $\mathbf{V}$, of $\mathbf{V}^{a n}$, the Berkovich analytification of a quasi-projective algebraic variety $\mathbf{V}$. Among other things, they show that $\widehat{\mathbf{V}}$ 
admits a definable strong deformation retraction onto a $\boldsymbol{\Gamma}$-internal subset $\boldsymbol{\Sigma}$, where $\boldsymbol{\Gamma}$ is the value group. Since, the divisible ordered Abelian group $\boldsymbol{\Gamma}$ is the natural model-theoretic framework for piecewise linear geometry, this result implies that, without any smoothness assumption on $\mathbf{V}, \mathbf{V}^{\text {an }}$ is locally contractible and admits a strong deformation retraction onto a piecewise linear space.

Our first result regarding these questions is that stable domination in a model $K$ of $\mathrm{SCVH}_{p, e}$ is characterized, via prolongations, by stable domination in the algebraic closure of $K$ (Proposition 6.9). From this, we deduce the following result, using a description of definable closure obtained in Proposition 5.5:

Theorem $\mathbf{C}$ (Corollary 6.11). The theories $\mathrm{SCVH}_{p, e}$ and $\mathrm{SCVF}_{p, e}$ are metastable over the value group $\boldsymbol{\Gamma}$.

Secondly, we establish the analogue of an important technical result from [14]. Before we may state this result, we need to recall some notions. A pro-definable set in $U$ is a set of the form $\mathbf{D}=\lim _{i \in I} \mathbf{D}_{i}$, where $\left(\mathbf{D}_{i}\right)_{i \in I}$ is a projective system in the category of definable sets and $I$ is a small index set. If all the $\mathbf{D}_{i}$ and the transition maps are $C$-definable, $\mathbf{D}$ is called $C$-pro-definable. A pro-definable function is a (bounded) family of definable functions (equivalently a function whose graph is pro-definable). The $(C$-)pro-definable sets form a category with respect to $\left(C\right.$-)pro-definable maps. If the pro-definable set $\mathbf{D}=\lim _{i \in I} \mathbf{D}_{i}$ is isomorphic to a pro-definable set with surjective transition functions, it is called strict prodefinable. Equivalently, for every $i \in I$, the set $\pi_{i}(\mathbf{D}) \subseteq \mathbf{D}_{i}$ is definable (and not just type-definable). Here, $\pi_{i}$ denotes the projection map on the $i$ th coordinate. Dually, one defines (strict) ind-definable sets. We refer to [14, Section 2.2] for the basic properties of these notions.

Let $\mathbf{X}$ be a $C$-definable set in ACVF. In [14] it is shown that there is a strict $C$-pro-definable set $\widehat{\mathbf{X}}$ such that for any $A \supseteq C$, the set $\widehat{\mathbf{X}}(A)$ is in canonical bijection with the set of $A$-definable global stably dominated types $p(x)$ such that $p(x) \vdash x \in \mathbf{X}$. If this is the case in a theory $T$, we say (rather loosely) that the set of stably dominated types in $T$ is strict pro-definable. From the proof in [14], we extract an abstract condition on a metastable NIP theory $T$ which implies that the set of stably dominated types is strict pro-definable in $T$. This yields the following:

Theorem D (Corollaries 7.7 and 7.8). The set of stably dominated types is strict pro-definable in $\mathrm{SCVH}_{p, e}$ as well as in $\mathrm{VDF}_{\mathcal{E C}}$.

The paper is organized as follows. In Section 2 we gather some preliminaries about valued fields, the model theory of algebraically closed valued fields, separably closed fields as well as separably closed valued fields. We then present, in Section 3 , the density theorem for semi-algebraic sets, and we prove Theorem A, namely that $\mathrm{SCVF}_{p, e}$ eliminates imaginaries down to the geometric sorts. Section 4 is devoted to the proof of Theorem B and of the fact that the geometric sorts classify the imaginaries even when working with Hasse derivations. This section mostly consists in the proof of a quantifier elimination result, of independent interest, for dense pairs of valued fields.

Subsequently, we show that various notions in $\mathrm{SCVH}_{p, e}$ reduce in the nicest possible way to $\mathrm{ACVF}_{p, p}$. In the short Section 5, we establish this for the definable and the algebraic closure; Section 6 gives a complete description of the stable stably embedded sets in $\mathrm{SCVH}_{p, e}$ (they are more or less the same as in $\mathrm{ACVF}_{p, p}$ ) as well as of the stably dominated types, in terms of stable domination of the prolongation in 
$\mathrm{ACVF}_{p, p}$. Putting all this together, we obtain the metastability of $\mathrm{SCVH}_{p, e}$ (Theorem C). In a final section we present an abstract framework, for metastable NIP theories, which guarantees the strict pro-definability of the set of stably dominated types, and we show that both $\mathrm{SCVH}_{p, e}$ and $\mathrm{VDF}_{\mathcal{E C}}$ fall under this framework, thus establishing Theorem D.

1.1. Acknowledgments. This collaboration began during the Spring 2014 MSRI program Model Theory, Arithmetic Geometry and Number Theory. The authors would like to thank MSRI for its hospitality and stimulating research environment.

We are grateful to the referee for a thorough reading of our paper, and for many useful suggestions.

\section{Preliminaries}

Let us fix some notation. We will normally denote (ind-,pro-) definable sets in a given theory by bold letters (such as $\mathbf{X}$ ). As customary, we will often identify such objects with their set of realizations in a universal domain (a fixed sufficiently saturated model), which we keep unspecified. In such cases, by a set (of parameters) we will mean a small subset of this universal domain.

Whenever $\mathbf{X}$ is a definable set (or a union of definable sets) and $A$ is a set of parameters, $\mathbf{X}(A)$ will denote $\mathbf{X} \cap A$. Usually in this notation there is an implicit definable closure, but we want to avoid that here because more often than not there will be multiple languages around and hence multiple definable closures which could be implicit. Similarly, if $\mathcal{S}$ is a set of definable sets, we will write $\mathcal{S}(A)$ for $\bigcup_{\mathbf{S} \in \mathcal{S}} \mathbf{S}(A)$. When the language is clear, we will write $A \leq K$ when $A$ is a substructure of $K$ (i.e., closed under function symbols).

We will be working with a fixed prime $p$, and will write $K_{p^{n}}$ for the set $\left\{x^{p^{n}} \mid\right.$ $x \in K\}$ of $p^{n}$-powers in a field $K$. Likewise, if $\mathbf{X}$ is a definable field, $\mathbf{X}_{p^{n}}$ is the definable set of $p^{n}$ powers. We write $K_{p^{\infty}}\left(\operatorname{resp} . \mathbf{X}_{p^{\infty}}\right)$ for the intersection of $K_{p^{n}}$ $\left(\mathbf{X}_{p^{n}}\right)$ over all $n$.

2.1. Imaginaries. Recall that, in model theory, an imaginary point is a class of a definable equivalence relation. To every theory $T$, we can associate a theory $T^{\mathrm{eq}}$ obtained by adding all the imaginary points. Every model $M$ of $T$ expands uniquely to a model $M^{\mathrm{eq}}$ of $T^{\mathrm{eq}}$. We write $\mathrm{dcl}^{\mathrm{eq}}$ and acl ${ }^{\mathrm{eq}}$ to denote the definable and algebraic closure in $M^{\mathrm{eq}}$.

Let $\mathbf{X}$ be a set definable with parameters in a sufficiently saturated and homogeneous structure $M$. We denote by $\ulcorner\mathbf{X}\urcorner \subseteq M^{\text {eq }}$ the set of points fixed by the group $G\ulcorner\mathbf{X}\urcorner$ of automorphisms stabilizing $\mathbf{X}$ globally. We say that $\mathbf{X}$ has a canonical parameter (or is coded) if it can be defined over $\ulcorner\mathbf{X}\urcorner \cap M$. Likewise, $\mathbf{X}$ has an almost canonical parameter (or is weakly coded) if it can be defined over acl ${ }^{\mathrm{eq}}(\ulcorner\mathbf{X}\urcorner) \cap M$, i.e., over some tuple with a finite orbit under the action of the same group. Note that if this finite orbit (viewed as a definable set) itself has a canonical parameter, then it is a canonical parameter for $\mathbf{X}$ as well.

A theory admits elimination of imaginaries precisely if every definable set has a canonical parameter.

\subsection{Valued fields.}


2.2.1. Notation and conventions. Whenever $(K$, val $)$ is a valued field, we will denote by $\boldsymbol{\Gamma}(K):=\operatorname{val}\left(K^{\star}\right)$ its value group, by $\mathcal{O}(K)$ its valuation ring, by $\mathfrak{m}(K)$ its maximal ideal, by $\mathbf{k}(K):=\mathcal{O}(K) / \mathfrak{m}(K)$ its residue ring and by $\operatorname{res}_{K}: \mathcal{O}(K) \rightarrow$ $\mathbf{k}(K)$ the canonical projection. When the field $K$ is clear from context, we will write $\Gamma, \mathcal{O}, \mathfrak{m}, k$ and res. We usually identify $\Gamma$ and $\Gamma_{\infty}:=\operatorname{val}(K)=\Gamma \cup\{\infty\}$. We will also denote by $\mathbf{V F}(K)$ the points of the valued field itself (this will make more sense once we consider multi-sorted structures).

Let us now briefly recall the geometric sorts from [8]. For $n \geq 1$, let $\mathbf{S}_{n}(K)$ be the set of $\mathcal{O}$-lattices in $K^{n}$, i.e., $\mathbf{S}_{n}(K) \simeq \mathbf{G L}_{n}(K) / \mathbf{G} \mathbf{L}_{n}(\mathcal{O})$. Note that, for any $s \in$ $\mathbf{S}_{n}(K)$, the quotient $s / \mathfrak{m} s$ is an $n$-dimensional $k$-vector space. One puts $\mathbf{T}_{n}(K):=$ $\dot{U}_{s \in \mathbf{S}_{n}(K)} s / \mathfrak{m} s$. Note that $\mathbf{T}_{n}(K)$ can similarly be identified with $\left(\mathbf{G L}_{n} / \mathbf{G}\right)(K)$, where $\mathbf{G}$ is the inverse image of the stabilizer of a non-zero vector under the residue map on $\mathbf{G L}_{n}(\mathcal{O})$. Hence it is indeed an imaginary sort. The map associating to an element of $\mathbf{T}_{n}$ the corresponding lattice in $\mathbf{S}_{n}$ is denoted by $\tau_{n}$.

When $n=1$, the map val : $\mathbf{G L}_{1}(K) \rightarrow \Gamma$ is a surjective homomorphism with kernel $\mathbf{G L}_{1}(\mathcal{O})$, and therefore $\mathbf{S}_{1} \cong \boldsymbol{\Gamma}$ canonically. Similarly, $\mathbf{k}^{\star}=(\mathcal{O} / \mathfrak{m})^{\star} \subseteq \mathbf{T}_{1}$ canonically. In fact $\mathbf{T}_{1}(K)$ is canonically isomorphic, as above, to the quotient of multiplicative groups $K^{\star} /(1+\mathfrak{m})$ which is often denoted $\mathbf{R V}(K)$.

2.2.2. The valuation topology. Let $\widetilde{T}$ be a theory of valued fields, with valued field sort VF. We consider $\mathbf{V F}^{n}$ with the definable valuation topology, where a definable subset $\mathbf{X}$ is open if each of its points belongs to a product of open balls contained in $\mathbf{X}$. Here, $\mathbf{X}$, the balls and the points are over parameters, but if $\mathbf{X}$ is open and over $K$, and $a \in \mathbf{X}(K)$ for some valued field $K$, then the ball can also be chosen over $K$. Continuous definable functions and other topological notions are defined similarly.

Given a valued field $K$, the collection $\mathbf{X}(K)$, where $\mathbf{X}$ ranges over $K$-definable open subsets of $\mathbf{V F}{ }^{n}$, forms a basis for the usual valuative topology on $K^{n}$. Note that an inclusion of valued fields $K \subseteq L$ is continuous if and only if $\Gamma(K)$ is a cofinal subset of $\boldsymbol{\Gamma}(L)$.

Lemma 2.1. Assume that $K$ is dense in $L$ in the valuation topology. Then we have $\mathbf{S}_{n}(K)=\mathbf{S}_{n}(L)$ and $\mathbf{T}_{n}(K)=\mathbf{T}_{n}(L)$ for every $n \geq 1$. In particular, the extension $L / K$ is immediate.

Proof. Since $K$ is dense in $L$, the set $K^{N}$ is dense in $L^{N}$ for every $N$, and so $\mathbf{G L}_{n}(K)$ is dense in $\mathbf{G L}_{n}(L)$, as $\mathbf{G} \mathbf{L}_{n}(L)$ is an open subset of $L^{n^{2}}$. For any $M \in \mathbf{G L}_{n}(L)$, the set $M \cdot \mathbf{G L}_{n}(\mathcal{O}(L))$ is open in $\mathbf{G L}_{n}(L)$, so it contains some $M_{0} \in \mathbf{G L}_{n}(K)$, showing that $\mathbf{S}_{n}(K)=\mathbf{G L}_{n}(K) / \mathbf{G L}_{n}(\mathcal{O}(K))=\mathbf{G L}_{n}(L) / \mathbf{G L}_{n}(\mathcal{O}(L))=\mathbf{S}_{n}(L)$.

Now let $t \in \mathbf{T}_{n}(L)$. By the previous paragraph, we know that $\tau_{n}(t)=s \in$ $\mathbf{S}_{n}(K)$, so applying a $K$-linear change of variables we may assume that $s=\mathcal{O}^{n}$ and $t \in \mathbf{k}(L)^{n}=\mathcal{O}(L)^{n} / \mathfrak{m} \mathcal{O}(L)^{n}$. But then $\pi^{-1}(t)$ is an open subset of $\mathcal{O}(L)^{n}$, so by the density assumption there is a tuple $\bar{a} \in \mathcal{O}(K)^{n}$ such that $\pi(\bar{a})=t$. It follows that $t \in \mathbf{T}_{n}(K)$.

Note that there are immediate extensions $L / K$ such that $K$ is not dense in $L$, e.g., the Puiseux series field $K=\bigcup_{n \in \mathbb{N}} \mathbb{C}\left(\left(t^{1 / n}\right)\right)$ inside the Hahn series field $L=\mathbb{C}\left(\left(t^{\mathbb{Q}}\right)\right)$. 
Our goal now is to show that every smooth subvariety of affine space (viewed as a definable subset of $\mathbf{V} \mathbf{F}^{n}$ ) is a topological manifold, i.e., definably locally homeomorphic to an open subset of $\mathbf{V F}^{m}$ for some $m$. If $\mathbf{X}$ is a variety over a field $K_{0}$, and $a \in \mathbf{X}\left(K_{0}\right)$, by a local coordinate system around $a$ we mean an étale map from a Zariski neighborhood of $a$ to $\mathbf{V F}^{d}$, all over $K_{0}$, taking $a$ to 0 (here $d$ is the dimension of $\mathbf{X}$ at $a$ ). The following observation is well known, see, e.g., [20, Proposition 4.9] or [19, Proposition I.3.24].

Fact 2.2. If a is a smooth point of a variety $X$, it admits a system of local coordinates.

The following statement is essentially the implicit function theorem in the valuative setting.

Proposition 2.3. Let $\mathbf{X}$ be a smooth subvariety of affine space, viewed as a definable subset of $\mathbf{V F}^{n}$ in a theory $\widetilde{T}$ of Henselian valued fields. Then the induced definable topology on $\mathbf{X}$ is the unique topology for which every local coordinate system around every point a of $\mathbf{X}$ is a homeomorphism in a neighborhood of a.

In particular, $\mathbf{X}$ admits a unique definable topology making $\mathbf{X}(K)$ locally homeomorphic to an open ball in $K^{d}$ around each point.

Proof. Uniqueness is clear by Fact 2.2. To show that every local coordinate system is a local homeomorphism, we first note that the problem is local for the Zariski topology on $\mathbf{X}$, and by [19, Prop. I.3.24] we may therefore assume that $\mathbf{X}$ is the zero set of a polynomial map $P\left(y_{1}, \ldots, y_{n}\right)=\left(f_{d+1}(\bar{y}), \ldots, f_{n}(\bar{y})\right): \mathbf{V F}^{n} \rightarrow \mathbf{V F}^{n-d}$, where the tangent space of $\mathbf{X}$ at $a$ is given as the kernel of $d P(a)$, and has dimension $d$.

Also, we are given a coordinate system $\bar{F}=\left(\bar{f}_{1}, \ldots, \bar{f}_{d}\right): \mathbf{X} \rightarrow \mathbf{V F}^{d}$, which we may assume to be globally étale. Let $F=\left(f_{1}, \ldots, f_{d}\right)$ be any lift of $\bar{F}$ to a polynomial function on $\mathbf{V F}^{n}$. Then $d F$ restricts to a bijection from the tangent space of $\mathbf{X}$ at $a$ to $\mathbf{V} \mathbf{F}^{d}$. It follows that the Jacobian matrix $\left(\partial f_{i} / \partial y_{j}\right)$ of the combined map $(F, P)$ is invertible.

By rescaling, we may assume that both $a$ and the coefficient of $F, P$ lie in $\mathcal{O}$. Replacing $\mathbf{X}$ by the graph of $F$, we are in the situation of Hensel's Lemma (as in, e.g., [17, Thm. 9.14] or [22, Thm. 7.4]). Thus, there is a value $r \in \boldsymbol{\Gamma}\left(K_{0}\right)$ (namely, the valuation of the Jacobian determinant at $a$ ), such that for any $\bar{x} \in \mathbf{V F}^{d}$ with $\operatorname{val}(\bar{x})>2 r$, there is a unique $\bar{y} \in \mathbf{X}$ with $F(\bar{y})=\bar{x}$ and $\operatorname{val}(\bar{y}-a) \geq \operatorname{val}(\bar{x})-r$. This inequality also gives the continuity.

Note that this shows, in particular, that there is a valuation topology on any smooth affine variety, independent of an embedding into affine space, and therefore that the same definition determines a topology on any (not necessarily affine) smooth variety.

2.3. Model theory of algebraically closed valued fields. Recall the following languages for valued fields:

- $\mathcal{L}_{\text {div }}=\mathcal{L}_{\text {ring }} \cup\{\operatorname{div}\}$ is the language with one sort VF, where $x \operatorname{div} y \Leftrightarrow$ $\operatorname{val}(x) \leq \operatorname{val}(y)$.

- $\mathcal{L}_{\Gamma}$ is the two-sorted language with sorts $\mathbf{V F}$ and $\boldsymbol{\Gamma}$, given by $\mathcal{L}_{\text {ring }}$ on $\mathbf{V F}$, $\mathcal{L}_{\text {oag }}=\{0,+,-,<, \infty\}$ on $\boldsymbol{\Gamma}$ and val $: \mathbf{V F} \rightarrow \boldsymbol{\Gamma}$. 
- $\mathcal{L}_{\Gamma k}$ is the three-sorted language with sorts $\mathbf{V F}, \mathbf{k}$ and $\boldsymbol{\Gamma}$, given by $\mathcal{L}_{\text {ring }}$ on $\mathbf{V F}$, (another copy of) $\mathcal{L}_{\text {ring }}$ on $\mathbf{k}, \mathcal{L}_{\text {oag }}$ on $\boldsymbol{\Gamma}$ and the functions val : VF $\rightarrow \boldsymbol{\Gamma}$ and Res : $\mathbf{V F}^{2} \rightarrow \mathbf{k}$ between the sorts. Here,

$$
\operatorname{Res}(x, y):=\left\{\begin{array}{l}
\operatorname{res}\left(\frac{x}{y}\right), \text { if } \infty \neq \operatorname{val}(y) \leq \operatorname{val}(x) ; \\
0, \text { otherwise. }
\end{array}\right.
$$

- $\mathcal{L}_{\mathrm{RV}}$ is the $\mathbf{R V}$-language (also called language with leading terms) with two sorts $\mathbf{V F}$ and $\mathbf{R V}$ where that last sort is interpreted as $\mathbf{V F}^{\star} /(1+\mathfrak{m})$. We add an element 0 to $\mathbf{R V}$ for the image of 0 . The language consists of the ring language on $\mathbf{V F}$, a map $\mathrm{rv}: \mathbf{V F} \rightarrow \mathbf{R V}$ and the language $\mathcal{L}_{\text {div }}$ on $\mathbf{R V}$. The symbol · interprets the (multiplicative) group structure on $\mathbf{R V}$ and the symbol + the trace of the addition when it is well defined. To be precise, if $\operatorname{val}(x)<\operatorname{val}(y)$, then $\operatorname{rv}(x)+\operatorname{rv}(y)=\operatorname{rv}(y)+\operatorname{rv}(x)=\operatorname{rv}(x)$, if $\operatorname{val}(x)=\operatorname{val}(y)=\operatorname{val}(x+y)$, then $\operatorname{rv}(x)+\operatorname{rv}(y)=\operatorname{rv}(x+y)$ and otherwise $\operatorname{rv}(x)+\operatorname{rv}(y)=0$. Finally, $\operatorname{rv}(x) \operatorname{div} \operatorname{rv}(y)$ is interpreted as $\operatorname{val}(x) \leq \operatorname{val}(y)$.

Note that we will write $\sum_{i} x_{i}$ where $x_{i} \in \mathbf{R V}$. This notation is slightly abusive as + as defined above is not associative. What we mean by $\sum_{i} x_{i}$ is in fact $\sum_{i \in I_{0}} x_{i}$ where $I_{0}$ is the set of indices $i$ such that $x_{i}$ is of minimal valuation.

- $\mathcal{L}_{\mathcal{G}}$ is the language in the geometric sorts (or geometric language) from $[8$, Section 3.1.], with set of sorts $\mathcal{G}:=\{\mathbf{V F}, \mathbf{k}, \boldsymbol{\Gamma}\} \cup\left\{\mathbf{S}_{n} \mid n \geq 1\right\} \cup\left\{\mathbf{T}_{n} \mid n \geq 1\right\}$. It is an extension of $\mathcal{L}_{\Gamma k}$. We use the notation from [8], although we write the value group additively and not multiplicatively.

Fact 2.4. The theory ACVF of algebraically closed non-trivially valued fields eliminates quantifiers in either of the languages $\mathcal{L}_{\text {div }}, \mathcal{L}_{\Gamma}, \mathcal{L}_{\Gamma k}, \mathcal{L}_{\mathrm{RV}}$ and $\mathcal{L}_{\mathcal{G}}$.

For the first three languages, we refer to [8, Theorem 2.1.1]. (In the case of $\mathcal{L}_{\text {div }}$, the result is more or less due to Robinson.) The quantifier elimination result in the language $\mathcal{L}_{\mathcal{G}}$ is [8, Theorem 3.1.2]. Quantifier elimination in $\mathcal{L}_{\mathrm{RV}}$ for ACVF is folklore and we are not aware that any proof exists in the literature. Let us give a sketch of the proof.

Proof of Fact 2.4, $\mathcal{L}_{\mathrm{RV}}$ case. We have to show that given two models $M$ and $N$ of ACVF such that $N$ is $|M|^{+}$-saturated and given any isomorphism $f: A \rightarrow B$ between $\mathcal{L}_{\mathrm{RV}}$-substructures of $M$ and $N$, respectively, we can extend $f$ to $M$. First, one can check that $f$ can be extended to the closure of $A$ under inverses (both in $\mathbf{V F}$ and $\mathbf{R V}$ ).

Let $a \in \mathbf{R V}(A)$ be such that there is no $c \in \mathbf{V F}(A)$ with $\operatorname{val}(c)=\operatorname{val}_{\mathbf{R V}}(a)$ where val $_{\mathbf{R V}}$ is induced by val on $\mathbf{R V}$. If $\operatorname{val}_{\mathbf{R V}}(a) \in \mathbb{Q} \otimes \operatorname{val}(A)$, let $n$ be minimal positive such that $n \operatorname{val}_{\mathbf{R V}}(a)=\operatorname{val}(e)$ for some $e \in \mathbf{V F}(A)$. There exists $c \in \mathbf{V F}(M)$ such that $c^{n}=e$ and $\operatorname{rv}(c)=a$. To show that this holds, it suffices to prove it for $e=1$ and that can be done easily by applying Hensel's lemma and the Frobenius on the residue field (if the residue characteristic is positive). Similarly there exists $d \in \mathbf{V F}(N)$ such that $d^{n}=f(e)$ and $\operatorname{rv}(d)=f(\operatorname{rv}(a))$. If $\operatorname{val}_{\mathbf{R V}}(a) \notin \mathbb{Q} \otimes \operatorname{val}(A)$, take any $c$ such that $\operatorname{rv}(c)=a$ and any $d \in \mathbf{V F}(N)$ such that $\operatorname{rv}(d)=f(\operatorname{rv}(a))$. Then one can extend $f$ by sending $c$ to $d$.

Repeating this last step, we may assume that $\operatorname{val}(\mathbf{V F}(A))=\operatorname{val}_{\mathbf{R V}}(\mathbf{R V}(A))$ (and that $\mathbf{R V}(A)$ and $\mathbf{V F}(A)$ are closed under inverses). Given $r \in \mathbf{R V}(A)$, let $a \in \mathbf{V F}(A)$ be an element with $\operatorname{val}(a)=\operatorname{val}_{\mathbf{R V}}(r)$. Then $\frac{r}{\operatorname{rv}(a)}$ is a well defined 
element $c$ of $\mathbf{k}(A)$, so $r=c \operatorname{rv}(a)$, and $f(r)$ is uniquely determined. Hence such an $f$ is completely determined by its reduct to $\mathcal{L}_{\Gamma k}$ (actually the sort $\boldsymbol{\Gamma}$ is useless here, but the two sorted language with $\mathbf{V F}$ and $\mathbf{k}$ is not usually considered) and so $f$ extends to $M$ by quantifier elimination in $\mathcal{L}_{\Gamma k}$.

We note that in the multi-sorted languages $\mathcal{L}_{\Gamma}, \mathcal{L}_{\Gamma k}, \mathcal{L}_{\mathrm{RV}}$ and $\mathcal{L}_{\mathcal{G}}$, all added sorts are interpretable in $\mathcal{L}_{\text {div }}$, and the structure is just the one induced by the corresponding interpretations in $\mathcal{L}_{\text {div }}$.

The completions of ACVF are given by specifying the pair of characteristics $(\operatorname{char}(\mathbf{V F}), \operatorname{char}(\mathbf{k})) \in\{(0,0),(0, p),(p, p) \mid p$ a prime number $\}$. The completion corresponding to $(p, q)$ is denoted by $\operatorname{ACVF}_{p, q}$.

The following is the main result of [8].

Fact 2.5 ([8, Theorem 3.4.10]). The theory ACVF eliminates imaginaries in $\mathcal{L}_{\mathcal{G}}$.

\subsection{Separably closed fields.}

2.4.1. Notation and conventions. Let $K$ be a field of characteristic $p>0$. Let $b=\left(b_{j}\right)_{j \in J}$ be a (possibly infinite) tuple from $K$ and let $I: J \rightarrow p=\{0, \ldots, p-1\}$ be a function with finite support (that is a function that has value 0 outside of a finite set). We denote by $b^{I}$ the monomial $\prod_{j \in J} b_{j}^{I(j)}$. The tuple $b$ is said to be a $p$-basis of $K$ if the monomials $b^{I}$ form a linear basis of $K$ as a vector space over $K_{p}$. Then every $x \in K$ can be uniquely written as $x=\sum_{I} x_{I}^{p} b^{I}$. The $x_{I}$ are called the $p$-components of $x$ (with respect to $b$ ) and the functions $f_{I}: K \rightarrow K$ sending $x$ to $x_{I}$ are called the $p$-coordinate functions or $\lambda$-functions. Any characteristic $p$ field admits a $p$-basis and all $p$-bases of $K$ have the same cardinality $e$, usually called the imperfection degree or the Ershov invariant of $K$. Obviously, we have $\left[K: K_{p}\right]=p^{e}$ when $e$ is finite.

A field $K$ is said to be separably closed if it has no proper separable algebraic extension. For a prime $p$ and $e<\infty$, let $\mathcal{L}_{p, e}^{\lambda}:=\mathcal{L}_{\text {ring }} \cup\left\{b_{1}, \ldots, b_{e}\right\} \cup\left\{f_{I} \mid I \in p^{e}\right\}$ be the language with one sort $\mathbf{K}$ and let $\mathrm{SCF}_{p, e}$, be the theory of characteristic $p$ separably closed fields with imperfection degree $e$, where the $b_{j}$ form a $p$-basis with corresponding $p$-coordinate functions given by the $f_{I}$. We will denote by $\lambda^{n}: \mathbf{K} \rightarrow \mathbf{K}^{p^{n e}}$ the definable function whose coordinates are the $f_{I_{n}} \circ \cdots \circ f_{I_{1}}$ for all tuples $\left(I_{n}, \ldots, I_{1}\right)$. Note that $\lambda^{n}(x)^{p^{n}}$ is the tuple of $\mathbf{K}_{p^{n}}$-coordinates of $x$ in the basis $b^{\left(I_{n}, \ldots, I_{1}\right)}=\prod_{j}\left(b^{I_{j}}\right)^{p^{j}}$.

Fact 2.6. The theory $\mathrm{SCF}_{p, e}$, eliminates quantifiers and imaginaries and is complete. In case $e>0$, it is stable not superstable.

The quantifier elimination result in that particular language is due to Delon $[3,4]$. The completeness result goes back to work of Ershov [7]. Stability and non superstability are proved in [29]. Elimination of imaginaries is due to Delon and a proof can be found in [4, Proposition 3.9].

The (type-definable) subfield $\mathbf{K}_{p \infty}$ is the largest perfect subfield of $\mathbf{K}$, and it is algebraically closed. The induced structure on $\mathbf{K}_{p^{\infty}}$ (by the ambient structure) is that of a pure algebraically closed field.

2.5. Hasse derivations. The $\lambda$-functions already give a "field with operator" flavor to separably closed fields. Separably closed fields can also be naturally equipped with more classical operators: Hasse derivations. As explained by Hoffmann [10], 
there are two natural ways in which to endow a separably closed field with Hasse derivations. Here, we follow Ziegler [30].

A Hasse derivation on a ring $R$ is a sequence $D=\left(D_{n}\right)_{n \in \mathbb{N}}$ of additive functions $D_{n}: R \rightarrow R$ such that for all $x, y \in R, D_{0}(x)=x$ and $D_{n}(x y)=$ $\sum_{k+l=n} D_{k}(x) D_{l}(y)$. We say that $D$ is iterative if $D_{m} \circ D_{n}=\left(\begin{array}{c}m+n \\ n\end{array}\right) D_{m+n}$ also holds. We will assume all Hasse derivations to be iterative.

Let $e \in \mathbb{N}$. Let $K$ be a field of characteristic $p>0$ and $\left(D_{1}, \ldots, D_{e}\right)$ be a tuple of commuting Hasse derivations on $K$ (i.e., $D_{i, n} \circ D_{j, m}=D_{j, m} \circ D_{i, n}$ for all $i, j \leq e$ and $n, m \in \mathbb{N})$. It is easy to check that $K_{p \infty}$ is contained in the field

$$
C_{\infty}:=\left\{x \in K \mid D_{i, n}(x)=0 \text { for all } i \leq e \text { and } n>0\right\}
$$

of (absolute) constants.

The field $K$ is said to be strict if

$$
K_{p}=C_{1}:=\left\{x \in K \mid D_{i, 1}(x)=0 \text { for all } i \leq e\right\} .
$$

(We then have $K_{p^{\infty}}=C_{\infty}$ ). Let

$$
\mathcal{L}_{p, e}^{D}:=\mathcal{L}_{\text {ring }} \cup\left\{D_{i, n} \mid 0<i \leq e \text { and } n \in \mathbb{N}\right\}
$$

and let $\mathrm{SCH}_{p, e}$, be the theory of characteristic $p$ separably closed strict fields of imperfection degree $e$ with $e$ commuting Hasse derivations $D_{i}=\left(D_{i, n}\right)_{n \in \mathbb{N}}$.

For $N \in \mathbb{N}^{e}$, we will denote $D_{N}(x)=D_{1, n_{1}} \circ \ldots \circ D_{e, n_{e}}(x)$ and $D_{\omega}(x)=$ $\left(D_{N}(x)\right)_{N \in \mathbb{N} e}$.

Note that any separably closed field of imperfection degree $e$ (and $p$-basis $b$ ) can be made into a strict field with $e$ commuting Hasse derivations by setting

$$
D_{i, n}\left(b^{I}\right)=\left(\begin{array}{c}
I(i) \\
n
\end{array}\right) b_{i}^{I(i) n} \prod_{j \neq i} b_{j}^{I(j)}
$$

and

$$
D_{i, n}(x)=\sum_{I} \lambda_{I}^{m}(x)^{p^{m}} D_{i, n}\left(b^{I}\right)
$$

for any $m$ such that $n<p^{m}$. For all $n>0$, we then have $D_{i, n}\left(b_{j}\right)=1$ if $i=j$ and $n=1$ and $D_{i, n}\left(b_{j}\right)=0$ otherwise. Such a $p$-basis is said to be canonical. Conversely, if $b$ is a canonical $p$-basis, the $D_{i, n}$ can be expressed as above using $b$ and $\lambda$.

Fact 2.7 ([30]). The theory $\mathrm{SCH}_{p, e}$ eliminates quantifiers and imaginaries and is complete.

As noted in [30], the quantifier elimination result can be deduced from quantifier elimination in $\mathrm{SCF}_{p, e}$. This remains true in the valued setting, as will be seen below.

2.6. Separably closed valued fields. We now consider a separably closed field $K$ of positive characteristic $p$ and finite imperfection degree $e$ endowed with a nontrivial valuation val. As is the case for algebraically closed valued fields, there is a number of natural languages in which to consider these structures:

- The one sorted language $\mathcal{L}_{\text {div }, p, e}^{\lambda}:=\mathcal{L}_{\text {div }} \cup \mathcal{L}_{p, e}^{\lambda} ;$

- The two sorted language $\mathcal{L}_{\Gamma, p, e}^{\lambda}:=\mathcal{L}_{\Gamma} \cup \mathcal{L}_{p, e}^{\lambda}$;

- The three sorted language $\mathcal{L}_{\Gamma k, p, e}^{\lambda}:=\mathcal{L}_{\Gamma k} \cup \mathcal{L}_{p, e}^{\lambda}$;

- The leading term language $\mathcal{L}_{\mathrm{RV}, p, e}^{\lambda}:=\mathcal{L}_{\mathrm{RV}} \cup \mathcal{L}_{p, e}^{\lambda}$ 
- The geometric language $\mathcal{L}_{\mathcal{G}, p, e}^{\lambda}:=\mathcal{L}_{\mathcal{G}} \cup \mathcal{L}_{p, e}^{\lambda}$.

Let $\mathrm{SCVF}_{p, e}$ denote the theory of separably closed non-trivially valued fields of characteristic $p$ and imperfection degree $e$ (with $\lambda$-functions) in either of these languages. Similarly we define $\mathcal{L}_{\mathrm{div}, p, e}^{D}, \mathcal{L}_{\Gamma, p, e}^{D}, \mathcal{L}_{\Gamma k, p, e}^{D}, \mathcal{L}_{\mathrm{RV}, p, e}^{D}$ and $\mathcal{L}_{\mathcal{G}, p, e}^{D}$ to be the languages with $e$ Hasse derivations and we denote by $\mathrm{SCVH}_{p, e}$ the theory (in any of these languages) of separably closed strict non-trivially valued fields of imperfection degree $e$ with $e$ commuting Hasse derivations. We will also denote by SCVF the theory of separably closed non-trivially valued fields in the language $\mathcal{L}_{\text {div }}$.

Proposition 2.8. Let $K$ be a separably closed non-trivially valued field. For all $n \in \mathbb{N}, K_{p^{n}}$ is dense in $\bar{K}$. Moreover, if $K$ is $\omega$-saturated, then $K_{p^{\infty}}$ is dense in $\bar{K}$. In particular $\mathbf{S}_{n}\left(K_{p^{\infty}}\right)=\mathbf{S}_{n}(\bar{K})$ and $\mathbf{T}_{n}\left(K_{p^{\infty}}\right)=\mathbf{T}_{n}(\bar{K})$ in this case.

Proof. Cf. [11, Lemma 5.2.5 and Remark 5.2.6]. The statement about $K_{p \infty}$ follows by compactness and the one about the $\mathbf{S}_{n}$ and $\mathbf{T}_{n}$ is a consequence of Lemma 2.1

In [11], Hong proved that $\mathrm{SCVF}_{p, e}$ eliminates quantifiers in the language with two sorts. We now show that his proof generalizes to any of the five languages we have been considering. Let us also mention that Hong later proved, in [12], a stronger quantifier elimination result using "parametrized $\lambda$-functions" which also covers the case of separably closed valued fields of infinite Ershov invariant.

Proposition 2.9. $\mathrm{SCVF}_{p, e}$ eliminates quantifiers in the one, two and three sorted languages, the leading term language as well as in the geometric language.

In the following pages, let $\mathcal{L}$ denote any of the five languages $\mathcal{L}_{\text {div }}, \mathcal{L}_{\Gamma}, \mathcal{L}_{\Gamma k}, \mathcal{L}_{\mathrm{RV}}$ and $\mathcal{L}_{\mathcal{G}}$ and $\mathcal{L}^{\lambda}=\mathcal{L} \cup \mathcal{L}_{p, e}^{\lambda}$ the corresponding enrichment with $\lambda$-functions (for some fixed $p$ and $e$ ). The proof relies on one main technical tool, $\lambda$-resolutions:

Lemma 2.10 ([11, Definition-Proposition 5.2.2]). Let $M \models \mathrm{SCVF}_{p, e}, A \leq M$ be a substructure and $\mathbf{X} \subset \mathbf{V F}{ }^{m}$ be quantifier free $\mathcal{L}^{\lambda}(A)$-definable. There exists $n \in \mathbb{N}$ such that $\lambda^{n}(\mathbf{X})=\mathbf{Y}$ for some quantifier free $\mathcal{L}(A)$-definable set $\mathbf{Y} \subseteq \mathbf{V F}^{m p^{e n}}$. Such a set $\mathbf{Y}$ is called a $\lambda$-resolution of $\mathbf{X}$.

Note that once we know quantifier elimination, all definable subsets of $\mathbf{V F}^{n}$ will have a $\lambda$-resolution.

Proof. This is an immediate consequence of the fact that $\lambda(x+y)$ and $\lambda(x y)$ can be written as polynomials in $\lambda(x)$ and $\lambda(y)$, and that $\lambda^{n}$ is onto.

Lemma 2.11. Let $M$ and $N$ be two non-trivially valued fields (considered as $\mathcal{L}$ structures), $A \leq M, f: A \rightarrow N$ be an $\mathcal{L}$-embedding and $a \in \mathbf{V F}(M)$ be transcendental over $\mathbf{V F}(A)$. Assume that $N$ is $|A|^{+}$-saturated and $\mathbf{V F}(N)$ is dense in $\overline{\mathbf{V F}(N)}$. Then $f$ can be extended to an $\mathcal{L}$-embedding $A(a) \rightarrow N$.

Before giving the proof, we mention that in ACVF, any definable function from an imaginary geometric sort to $\mathbf{V F}$ has finite image. This follows for example from the fact that there are uncountable models of ACVF whose imaginary part is countable (along with quantifier elimination for ACVF). See Lemma 5.3 for a similar argument for SCVF or SCVH.

Proof. By compactness, it suffices to prove that for every quantifier free $\mathcal{L}(A)$ definable set $\mathbf{X}$ such that $a \in \mathbf{X}(M), f_{\star} \mathbf{X} \cap \mathbf{V F}(N) \neq \emptyset$. Since $N$ is non-trivially valued, $\overline{\mathbf{V F}(N)}$ is a model of ACVF, and since ACVF eliminates quantifiers in $\mathcal{L}$, 
$f_{\star} \mathbf{X} \cap \overline{\mathbf{V F}(N)} \neq \emptyset$. If $f_{\star} \mathbf{X} \cap \overline{\mathbf{V F}(N)}$ has non empty interior, then we conclude by density of $\mathbf{V F}(N)$ in $\overline{\mathbf{V F}(N)}$. If it has empty interior, then $f_{\star} \mathbf{X} \cap \overline{\mathbf{V F}(N)}$ is finite and so is $\mathbf{X} \cap \overline{\mathbf{V F}(M)}$. In particular it follows that $a$ is algebraic (in ACVF) over $A$ and hence (by the remark preceding the proof) over $\mathbf{V F}(A)$. This contradicts the fact that $a$ is transcendental over $\mathbf{V F}(A)$.

In fact, $f(a)$ can be chosen in any dense subfield $K_{0}$ of $\mathbf{V F}(N)$.

Corollary 2.12. Let $M$ and $N$ be two non-trivially valued fields (considered as $\mathcal{L}$-structures), $A \leq M, f: A \rightarrow N$ be an $\mathcal{L}$-embedding. Assume that $N$ is separably closed and $|M|^{+}$-saturated. Let $\mathbf{V F}(A) \leq K_{0} \leq \mathbf{V F}(M)$ be such that the extension $\mathbf{V F}(A) \leq K_{0}$ is separable. Then $f$ can be extended to an $\mathcal{L}$-embedding $A\left(K_{0}\right) \rightarrow N$.

Proof. First, if $K_{0}$ is an algebraic extension of $\mathbf{V F}(A)$, then, by quantifier elimination in ACVF, $f$ extends to an $\mathcal{L}$-embedding of $A\left(K_{0}\right)$ into $N$ (a priori, the image of $f$ is in $\bar{N}$, but because $N$ is separably closed, it is, in fact, in $N$ ). So we may always assume that $\mathbf{V F}(A)$ is separably closed.

By Proposition 2.8, $N$ is dense in $\bar{N}$. By compactness and saturation of $N$, it is enough to show the result for $K_{0}$ which is a finitely generated separable extension of $\mathbf{V F}(A)$. Note that such an extension admits a separating transcendence basis. Using Lemma 2.11, we may thus conclude by induction on $\operatorname{trdeg}\left(K_{0} / \mathbf{V F}(A)\right)$.

Proof of Proposition 2.9 (following [11, Proof of 5.2.1, p.59]). Let $M, N$ be two models of $\mathrm{SCVF}_{p, e}, A \leq M$ and $f: A \rightarrow N$ an $\mathcal{L}^{\lambda}$-embedding. We have to show, given $a \in M$ and provided $N$ is $|M|^{+}$-saturated, that $f$ extends to $A(a)$. By compactness, it suffices to show that for every quantifier free $\mathcal{L}^{\lambda}(A)$-definable set $\mathbf{X}$ such that $a \in \mathbf{X}(M), f_{\star} \mathbf{X} \cap N \neq \emptyset$. By Lemma 2.10, we may assume that $\mathbf{X}$ is $\mathcal{L}(A)$ definable, at the cost of turning $a$ into a finite tuple of elements of $\operatorname{VF}(M)$. Note that, because $A$ is closed under $\lambda$-functions, that $\mathbf{V F}(M) / \mathbf{V F}(A)$ is a separable field extension, so we may extend $f$ to an $\mathcal{L}$-embedding $M \rightarrow N$ by Corollary 2.12. We then have $f(a) \in f_{\star} \mathbf{X} \cap N$ and that concludes the proof.

Corollary 2.13. Let $M \models \mathrm{SCVF}_{p, e}$. The theory of $M$ is completely determined by the $\mathcal{L}_{\mathrm{div}}$-isomorphism type of $\mathbb{F}_{p}\left[b_{1}, \ldots, b_{e}\right]$.

From quantifier elimination (Proposition 2.9), the existence of $\lambda$-resolutions and the fact that $\mathrm{ACVF}_{p, p}$ is NIP, we obtain the following.

Corollary 2.14 (Delon, see [11, Corollary 5.2.13]). Any completion of $\mathrm{SCVF}_{p, e}$ is NIP.

Similar results hold for $\mathrm{SCVH}_{p, e}$ :

Proposition 2.15. $\mathrm{SCVH}_{p, e}$ is complete and eliminates quantifiers in the one, two and three sorted languages, the leading term language as well as in the geometric language.

The proof uses the following notion:

Definition 2.16. Let $M \models \mathrm{SCVH}_{p, e}$ and $A \subseteq M$. A $p$-basis $b$ of $M$ is said to be very canonical over $A$ if it is a canonical $p$-basis such that $b_{i} \in \mathcal{O}$ for all $i$ and the res $\left(b_{i}\right)$ are algebraically independent over $\mathbf{k}(A)$.

Lemma 2.17. Let $M \models \mathrm{SCVH}_{p, e}$ and $A \subseteq M$. If $M$ is $|A|^{+}$-saturated, then $M$ admits a p-basis which is very canonical over $A$. 
Proof. By [30, Corollary 4.2], $M$ has a canonical $p$-basis. As $D_{i, n}\left(\mathbf{V F}_{p^{\infty}}\right)=0$ for any $n>0$, if $b$ is a canonical $p$-basis and $a$ is an $e$-tuple from $\mathbf{V F}_{p \infty}(M)$, then $b+a$ is also a canonical $p$-basis. Since $\mathbf{V F}_{p^{\infty}}(M)$ is dense in $\mathbf{V F}(M)$, for any $c \in \mathbf{V F}(M), \operatorname{res}\left(c+\mathbf{V F}_{p^{\infty}}(M)\right)=\mathbf{k}(M)$. Using $|A|^{+}$-saturation, one can find a tuple $c \in \mathbf{k}(M)$ algebraically independent over $\mathbf{k}(A)$ and a tuple $a \in \mathbf{V F}_{p^{\infty}}$ such that $\operatorname{res}(b+a)=c$.

Proof of Proposition 2.15. Let us first prove quantifier elimination in the leading term language. Quantifier elimination in the one, two and three sorted languages follow formally. To be exact, in the case of the three sorted language, a little work is still necessary as there is no sort for $\boldsymbol{\Gamma}$ in $\mathcal{L}_{\mathrm{RV}}$. But, when doing a back and forth, that can be easily taken care of by lifting points in $\boldsymbol{\Gamma}$ to $\mathbf{R V}$ using quantifier elimination in ACVF. We will be using Ziegler's trick from [30].

Let $\varphi(x)$ be an $\mathcal{L}_{\mathrm{RV}}^{D}$-formula. As noted above, the $D_{i, n}$ can be expressed in terms of $b$ and $\lambda$. It follows that $\varphi(x)$ is equivalent to an $\mathcal{L}_{\mathrm{RV}}^{\lambda}$-formula $\psi(x)$ and that by Proposition 2.9, we may assume that $\psi$ is quantifier free. Note that, by Corollary 2.13, the formula $\psi$ does not depend on the actual choice of very canonical p-basis.

The only occurrences of $b$ and $\lambda$ in $\psi$ are in terms of the form $\operatorname{rv}\left(\sum_{I} P_{I}(x) b^{I}\right)$ where the $P_{I}$ and $Q_{I}$ are polynomial in $\lambda^{m}(x)$ for some $m \in \mathbb{N}$ (provided we rewrite $\sum_{I} P_{I}(x) b^{I}=0$ into $\left.\operatorname{rv}\left(\sum_{I} P_{I}(x) b^{I}\right)=0\right)$. Applying the Frobenius automorphism $m$-times, we may assume that the $P_{I}$ and $Q_{I}$ are polynomials in $\lambda^{m}(x)^{p^{m}}$. But by [30, Lemma 4.3], the $\lambda^{m}(x)^{p^{m}}$ can be expressed as polynomials in the $D_{i, n}(x)$ and $b$. We may therefore assume that the $P_{I}$ and $Q_{I}$ are polynomials in the $D_{i, n}(x)$.

Taking $b$ to be a very canonical basis over $\langle x\rangle$, the structure generated by $x$ (which we can do because the choice of very canonical $p$-basis did not matter so far), we get that

$$
\mathrm{rv}\left(\sum_{I} P_{I}(x) b^{I}\right)=\sum_{I} \operatorname{rv}\left(P_{I}(x)\right) \operatorname{res}(b)^{I} .
$$

Moreover, for all $t_{I} \in \operatorname{rv}(\langle x\rangle), \sum_{I} t_{I} \operatorname{res}\left(b^{I}\right)=0$ if and only if $\bigwedge_{I} t_{I}=0$. Therefore, $\psi$ can be rewritten so that $b$ does not appear in $\psi$ (and the rewriting does not depend on the point $x$ we are considering). This concludes the proof in the leading term language.

Let us now prove quantifier elimination in the geometric language. Let $M$ and $N$ be models of $\mathrm{SCVH}_{p, e}$ in the geometric language, $A \leq M$ and $f: A \rightarrow N$ an $\mathcal{L}_{\mathcal{G}}^{D}$-embedding. Assume that $M$ is $\omega$-saturated and $N$ is $|M|^{+}$saturated. We may assume that $\mathbf{V F}(A)$ is strict. Indeed, by [30, Lemma 2.4], there is a smallest strict extension of $\mathbf{V F}(A)$ which is uniquely determined up to isomorphism - as a field extension, it is algebraic and purely inseparable.

It now follows from [30, Corollary 2.2] that the field extension $\mathbf{V F}(A) \subseteq \mathbf{V F}(M)$ is separable. By Corollary 2.12, $f$ extends to an $\mathcal{L}_{\mathcal{G}}$-embedding of $M$ into $N$, so

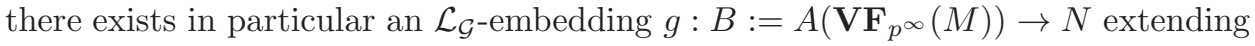
$f$. Clearly $g\left(\mathbf{V F}_{p^{\infty}}(M)\right) \subseteq \mathbf{V F}_{p^{\infty}}(N)$. Since we have added only absolute constants, $g$ is automatically an $\mathcal{L}_{\mathcal{G}}^{D}$-embedding. As $M$ is $\omega$-saturated, $\mathbf{V F}_{p \infty}(M)$ is dense in $\mathbf{V F}(M)$ by Proposition 2.8, and so $B$ is generated by $\mathbf{V F}(B)$. We may now use quantifier elimination in the one sorted language to extend $g$ to $M$. This concludes the proof in the geometric language. 
We will sometimes consider the set of all geometric sorts except the valued field sort VF. We will call these sorts the imaginary geometric sorts and write $\mathcal{G}^{\text {im }}:=$ $\mathcal{G} \backslash\{\mathbf{V F}\}=\{\boldsymbol{\Gamma}, \mathbf{k}\} \cup\left\{\mathbf{S}_{n} \mid n \geq 1\right\} \cup\left\{\mathbf{T}_{n} \mid n \geq 1\right\}$.

Corollary 2.18. Let $K \models T$ and $A \leq K$, where $T=\mathrm{SCVH}_{p, e}^{\mathcal{G}}$ (or $T=\mathrm{SCVF}_{p, e}^{\mathcal{G}}$, respectively). Let $L=\bar{K}$. Then $\mathcal{G}^{\operatorname{im}}(K)=\mathcal{G}^{\operatorname{im}}(L)$, and restriction to $K$-points determines an equivalence between the $\mathcal{L}_{\mathcal{G}}^{D}(A)$-definable sets (the $\mathcal{L}_{\mathcal{G}}^{\lambda}(A)$-definable sets, respectively) in the multi-sorted structure $\mathcal{G}^{\mathrm{im}}(K)$ and the $\mathcal{L}_{\mathcal{G}}(A)$-definable sets in $\mathcal{G}^{\mathrm{im}}(L)$.

Proof. We may assume that $T=\mathrm{SCVH}_{p, e}^{\mathcal{G}}$, as the result for $\mathrm{SCVF}_{p, e}^{\mathcal{G}}$ is a consequence of the one for $\mathrm{SCVH}_{p, e}^{\mathcal{G}}$. By Proposition 2.8, we have $\mathcal{G}^{\operatorname{im}}(K)=\mathcal{G}^{\operatorname{im}}(L)$. The statement about definable sets follows from quantifier elimination for $\mathrm{SCVH}_{p, e}$ in the language $\mathcal{L}_{\mathcal{G}}^{D}$ (Proposition 2.15).

Remark 2.19.

(1) Note that in $\mathrm{SCVF}_{p, e}$ or $\mathrm{SCVH}_{p, e}$, the field of absolute constants is not stably embedded (although, since $\mathbf{V F} \subseteq \operatorname{dcl}\left(\mathbf{V F}_{p^{n}}\right)$, each of the $\mathbf{V} \mathbf{F}_{p^{n}}$ is).

Indeed, let $M$ be an $\omega$-saturated model, $a \in \mathbf{V F}(M) \backslash \mathbf{V} \mathbf{F}_{p^{\infty}}(M)$, and let $B$ be the set of balls in $M$ that contain $a$. If $\mathbf{V F}_{p^{\infty}}(M)$ were stably embedded, then, by quantifier elimination, $B$ would be $\mathcal{L}_{\mathcal{G}}\left(\mathbf{V F}_{p^{\infty}}(M)\right)$ definable and by definable spherical completeness of ACVF, there would exist $c \in \bigcap_{b \in B} b\left(\mathbf{V F}_{p^{\infty}}(M)\right)$. But $\bigcap_{b \in B} b(M)=\{a\}$ and $a \notin \mathbf{V F}_{p^{\infty}}(M)$.

(2) Nevertheless, by quantifier elimination, $\mathbf{V} \mathbf{F}_{p \infty}$ is a pure algebraically closed valued field in the following (weak) sense: any definable set in $\mathbf{V F}_{p \infty}(M)$ (including the geometric sorts) is the intersection of a quantifier free $\mathcal{L}_{\mathcal{G}}(M)$ definable set with (some Cartesian power of) $\mathbf{V} \mathbf{F}_{p^{\infty}}$.

\section{IMAGINARIES AND DENSITY}

\subsection{The density theorem.}

Lemma 3.1. Let $\mathbf{V}$ be an irreducible variety defined over $K=\bar{K}^{\mathrm{sep}}$. Then any $\bar{K}$-definable open subvariety $\mathbf{U} \subseteq \mathbf{V}$ is defined over $K$.

Proof. We may suppose that $\operatorname{char}(K)=p>0$, and we may assume that $\mathbf{V}$ is affine and $\mathbf{U}=\mathbf{V}_{f}$, where $f \in \bar{K}[\mathbf{V}]=K[\mathbf{V}] \otimes_{K} \bar{K}$. So there is some $N=p^{k}$ such that $f^{N} \in K[\mathbf{V}]$. Thus, $\mathbf{V}_{f}=\mathbf{V}_{f^{N}}$ is defined over $K$.

The valuation topology on powers of a model L of ACVF determines, in the terminology of $[28,2.11]$, a topological system (using the language $\mathcal{L}_{\text {div }}$ ): All polynomials are continuous, and punctured balls are open. Further, it satisfies the assumptions of $[28,2.15]$, and therefore we have:

Fact $3.2([28,2.18])$. In $\mathrm{ACVF}$, a set $\mathbf{X} \subseteq \mathbf{V F}^{n}(L)$ definable with parameters is Zariski dense if and only if it contains a non-empty open ball.

Using Proposition 2.3, we obtain the same result for any smooth variety:

Corollary 3.3. A definable subset $\mathbf{X}$ of a (connected) smooth algebraic variety $\mathbf{V}$ in ACVF is Zariski dense if and only if it contains a non-empty subset which is open in $\mathbf{V}$ for the valuation topology. 
Proof. The claim is local, hence we may choose local coordinates as in Proposition 2.3 and reduce to the case $\mathbf{V}=\mathbf{V F}^{n}$. Now the claim follows from Fact 3.2.

Lemma 3.4. If $\mathrm{K} \models \mathrm{SCVF}$, and $\mathbf{X} \subseteq \mathbf{V F}^{n}$ is a semi-algebraic subset defined over $K$, then there are absolutely irreducible affine subvarieties $\mathbf{Y}_{i}$ of $\mathbf{V} \mathbf{F}^{n}$ defined over $K$ and $\mathbf{X}_{i} \subseteq \mathbf{Y}_{i}$ given by a Boolean combination of conditions of the form $\operatorname{val}(h)>0$, where $h$ is an invertible regular function on $\mathbf{Y}_{i}$, such that $\mathbf{X}=\bigcup_{i} \mathbf{X}_{i}$.

Proof. Let $J$ be the set of polynomials $F$ over $K$ such that for some polynomial $G$, $F \operatorname{div} G$ or $G \operatorname{div} F$ occurs in a semi-algebraic definition of $\mathbf{X}$, and for every subset $I$ of $J$, let $\mathbf{W}_{I}$ be the locally closed subset of $\mathbf{V F}^{n}$ given by

$$
\bigwedge_{F \in I} F(\bar{x})=0 \wedge \prod_{F \notin I} F(\bar{x}) \neq 0
$$

For each $I, \mathbf{W}_{I}$ is an affine subvariety of $\mathbf{V F}^{n}$, and $\mathbf{X} \cap \mathbf{W}_{I}$ is given by a Boolean combination of polynomial equations and valuative inequalities as in the definition. Hence, by restricting to $\mathbf{W}_{I}$, we may assume that $\mathbf{X}$ itself was given by such a Boolean combination to begin with.

Writing $\mathbf{X}$ in disjunctive normal form, we present $\mathbf{X}$ as a finite union of definable sets $\mathbf{X}_{i}$, each the intersection of a (Zariski) locally closed subset $\mathbf{Z}_{i}$ with valuative inequalities. Let $\mathbf{Y}_{i}$ be the Zariski closure of $\mathbf{X}_{i}(K)$ in $\mathbf{Z}_{i}$ (over $K$ ). The irreducible components of $\mathbf{Y}_{i}$ are defined over $K$, since $K$-points are dense. Hence we may assume that each $\mathbf{Y}_{i}$ is (absolutely) irreducible. Then they satisfy the requirements of the claim.

Proposition 3.5 (Hong [11, Theorem 5.3.1]). Let $\mathrm{K} \models \mathrm{SCVF}$ and $\mathbf{X} \subseteq \mathbf{V F}^{n}(K)$ be a semi-algebraic subset of $\mathbf{V F}^{n}(K)$. Let $L=\bar{K}$. Then there is a quantifier-free $\mathcal{L}_{\text {div }}(K)$-formula $\psi(\bar{x})$ with $\psi(K)=\mathbf{X}$ and such that $\psi(K)$ is dense in $\psi(L)$.

Remark 3.6. Actually, Hong states his result only for $\aleph_{1}$-saturated $K$, but it is easy to see that the result for general $K$ follows from this. We add a full proof for convenience.

Proof. By Lemma 3.4, we may assume that $\mathbf{X}$ is a (non-empty) valuation-open subset of a variety $\mathbf{V}$ over $K$. Since $\mathbf{X}$ is Zariski-dense in $\mathbf{V}$, we may pass to the smooth locus and assume that $\mathbf{V}$ is smooth. By Proposition 2.3, we may now reduce to the case of $\mathbf{V}=\mathbf{V F}^{d}$. Indeed, as $K$ is separably closed, there exists an open cover of $\mathbf{V}$ by subvarieties $\mathbf{V}_{i}$ and étale maps $f_{i}: \mathbf{V}_{i} \rightarrow \mathbf{V} \mathbf{F}^{d}$ defined over $K$ which are local homeomorphisms. As $K=\bar{K}^{\text {sep }}$ and $f_{i}$ is étale, for every $a \in \mathbf{V}_{i}(L)$ one has $f_{i}(a) \in \mathbf{V} \mathbf{F}^{d}(K)$ if and only if $a \in \mathbf{V}_{i}(K)$. Now $\mathbf{V} \mathbf{F}^{d}(K)$ is dense in $\mathbf{V} \mathbf{F}^{d}(L)$ by Proposition 2.8, and so $\mathbf{V}_{i}(K)$ is dense in $\mathbf{V}_{i}(L)$.

Corollary 3.7. Let $\mathrm{K} \models \mathrm{SCVF}$ and $\mathbf{X}$ be a semi-algebraic subset of $\mathbf{V F}^{n}(K)$. Let $\mathbf{V}$ be the Zariski closure of $\mathbf{X}$, and let $d=\operatorname{dim}(\mathbf{V})$. Then there is a semi-algebraic subset $\mathbf{X}^{\prime}$ of $\mathbf{X}$ and a polynomial map $f: \mathbf{X}^{\prime} \rightarrow \mathbf{V F}^{d}$ defined over $K$ which induces a homeomorphism between $\mathbf{X}^{\prime}$ and $\mathcal{O}^{d}(K)$.

Proof. The result follows from the proof of Proposition 3.5.

3.2. Elimination of imaginaries in $\mathrm{SCVF}_{p, e}$. In this section, $K$ denotes a sufficiently saturated and homogeneous model of $\operatorname{SCVF}_{p, e}$, and $L:=\bar{K}$ its algebraic closure, so a model of $\mathrm{ACVF}_{p, p}$. We consider $L$ in the language $\mathcal{L}_{\mathcal{G}}$, and $K$ in the language $\mathcal{L}_{\mathcal{G}, p, e}^{\lambda}$. In $K$, acl ${ }^{\text {eq }}$ etc. refers to $\mathrm{SCVF}_{p, e}^{\mathrm{eq}}$. 
Lemma 3.8. (1) Any automorphism of $K$ (uniquely) lifts to an automorphism of $L$. In particular, if $\bar{a}, \bar{b} \in \mathcal{G}(K)$ and $\bar{b} \in \operatorname{dcl}_{\mathrm{ACVF}_{p, p}}(\bar{a})$, then $\bar{b} \in$ $\operatorname{dcl}_{\mathrm{SCVF}_{p, e}}(\bar{a})$.

(2) For every tuple $\bar{a} \in \mathcal{G}(L)$ there is a tuple $\bar{a}^{\prime} \in \mathcal{G}(K)$ such that $\operatorname{dcl}_{\operatorname{ACVF}_{p, p}}(\bar{a})=$ $\operatorname{dcl}_{\mathrm{ACVF}_{p, p}}\left(\bar{a}^{\prime}\right)$.

(3) In the structure $K$, finite sets are coded, i.e., for every $\left\{\bar{a}_{1}, \ldots, \bar{a}_{n}\right\} \subseteq \mathcal{G}(K)$ there is $\bar{b} \in \mathcal{G}(K)$ which is interdefinable in $K^{\text {eq }}$ with $\left\ulcorner\left\{\bar{a}_{1}, \ldots, \bar{a}_{n}\right\}\right\urcorner$.

Proof. (1) is clear. To prove (2), note that $\mathbf{S}_{n}(K)=\mathbf{S}_{n}(L)$ and $\mathbf{T}_{n}(K)=\mathbf{T}_{n}(L)$ for every $n \geq 1$. So it is enough to show (2) for elements of the field sort. But for any $a \in L$ there is $m$ such that $a^{p^{m}} \in K$, and $a$ and $a^{p^{m}}$ are interdefinable.

(3) By elimination of imaginaries in $\mathrm{ACVF}_{p, p}$ down to the geometric sorts, it follows that finite subsets of $\mathcal{G}(L)$ are coded in $\mathcal{G}(L)$. We finish combining (2) and (1).

Theorem 3.9. The theory $\mathrm{SCVF}_{p, e}$ eliminates imaginaries down to the geometric sorts.

Proof. Let $\mathbf{X}$ be a definable set and $A$ contain $\mathcal{G}(\ulcorner\mathbf{X}\urcorner)$. We have to prove that $\mathbf{X}$ can be defined over $A$. Since, by Lemma 3.8, finite sets are coded, it is enough to show that $\mathbf{X}$ is weakly coded, so we may assume that $A$ is algebraically closed. Also, because $\mathbf{V F}$ is dominant, we may assume that $\mathbf{X}$ is a subset of some Cartesian power of VF. Let $K_{0}:=\mathbf{V F}(A)$. Since $K_{0}$ is closed under $\lambda$-functions and relatively algebraically closed in $K$, the extension $K / K_{0}$ is regular.

By Lemma 2.10, there exists an $n$ such that $\lambda^{n}(\mathbf{X})=\psi(K)$ where $\psi$ is a quantifier free $\mathcal{L}_{\text {div }}(K)$-formula. As $\lambda$ is injective and $\emptyset$-definable it follows that finding a (weak) code for $\lambda^{n}(\mathbf{X})$ is equivalent to finding one for $\mathbf{X}$, so we may assume that $\mathbf{X}=\psi(K)$ for some quantifier free $\mathcal{L}_{\text {div }}(K)$-formula $\psi(x)$. Moreover, by Proposition 3.5, we may assume that $\mathbf{X}$ is dense in $\mathbf{Y}:=\psi(L)$.

Let $\mathbf{V}=\overline{\mathbf{X}}^{Z a r}$. Then $\mathbf{V}$ is $K_{0}$-definable, by the existence of a smallest field of definition of $\mathbf{V}$. Moreover, $\mathbf{Y} \subseteq \mathbf{V}(L)$. We proceed by induction on $\operatorname{dim}(\mathbf{V})$. Since $\mathbf{V}(K)$ is Zariski-dense in $\mathbf{V}(L)$ and the extension $K / K_{0}$ is regular, the (absolute) irreducible components $\mathbf{V}_{1}, \ldots, \mathbf{V}_{l}$ of $\mathbf{V}$ are defined over $K_{0}$. Hence, encoding $\mathbf{V}_{i}(K) \cap \mathbf{X}$ one by one, we may assume that $\mathbf{V}$ is absolutely irreducible. It obviously suffices to encode $\mathrm{cl}_{\text {val }}^{K}(\mathbf{X})$ and $\mathrm{cl}_{\text {val }}^{K}(\mathbf{X}) \backslash \mathbf{X}$, where $\mathrm{cl}_{\text {val }}^{K}(\mathbf{X})$ denotes the valuative closure of $\mathbf{X}$ in $\mathbf{V F}^{n}(K)$. But $\mathrm{cl}_{\text {val }}^{K}(\mathbf{X}) \backslash \mathbf{X} \subseteq \mathrm{cl}_{\text {val }}^{L}(\mathbf{Y}) \backslash \mathbf{Y}$, a subset of $\mathbf{V}(L)$ which has

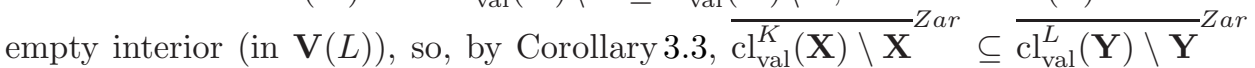
is a strict subvariety of $\mathbf{V}$. By induction $\mathrm{cl}_{\text {val }}^{K}(\mathbf{X}) \backslash \mathbf{X}$ is $A$-definable. It follows that we may assume $\mathbf{X}$ valuatively closed in $\mathbf{V}(K)$.

The set $\tilde{\mathbf{Y}}=\mathrm{cl}_{\text {val }}^{L}(\mathbf{Y})=\mathrm{cl}_{\text {val }}^{L}(\mathbf{X})$ is also definable by a quantifier free $\mathcal{L}_{\text {div }}(K)$ formula, say by $\widetilde{\psi}$, and one has $\widetilde{\psi}(K)=\mathrm{cl}_{\mathrm{val}}^{K}(\mathbf{X})=\mathbf{X}$. By elimination of imaginaries in $\operatorname{ACVF}$ (Fact 2.5), $\tilde{\mathbf{Y}}$ is definable over some $e \in \mathcal{G}(\ulcorner\widetilde{\mathbf{Y}}\urcorner)$ and, by Lemma 3.8, we may assume that $e \in \mathcal{G}(K)$. Clearly $\mathbf{X}$ is $\mathcal{L}_{\mathcal{G}}$-definable over $e$ (in $K$ ) so there only remains to show that $e \in\ulcorner\mathbf{X}\urcorner$. Let $\sigma$ be an automorphism of $K$ that stabilizes $\mathbf{X}$ globally, then the (unique) extension of $\sigma$ to $L$ must stabilize $\mathrm{cl}_{\text {val }}^{L}(\mathbf{X})=\widetilde{\mathbf{Y}}$ and hence fixes $e$. So $e \in\ulcorner\mathbf{X}\urcorner$. 


\section{IMAGINARIES, DEFINABLE TYPES AND DENSE PAIRS}

4.1. Quantifier elimination in dense pairs of valued fields. Much of the following is inspired by work of Delon [5].

4.1.1. The pure field case. Let $\mathcal{L}_{\mathrm{P}}$ denote the language $\mathcal{L}_{\text {ring }} \cup\left\{\mathbf{F}, \mathbf{l d}_{n}, \ell_{n, i} \mid n \in\right.$ $\mathbb{N}_{>0}$ and $\left.0<i \leq n\right\}$ where $\mathbf{F}$ is a new unary predicate, the $\mathbf{l d}_{n}$ are new $n$-ary predicate symbols and the $\ell_{n, i}$ are new $n+1$-ary function symbols. Note that, for this section, the field sort will be denoted by $\mathbf{K}$ and not by $\mathbf{V F}$, as it is not valued. Let $\mathrm{T}_{\mathrm{P}}$ be the $\mathcal{L}_{\mathrm{P}}$-theory of pairs of fields, with $\mathbf{F}$ defining the smaller field, and where $\mathbf{l d}_{n}\left(y_{1}, \ldots, y_{n}\right)$ holds if and only if the $y_{i}$ are linearly independent over $\mathbf{F}$ and if $\mathbf{l d}_{n}(y) \wedge \neg \mathbf{l d} \mathbf{d}_{n+1}(x, y)$ holds then $x=\sum_{i} \ell_{n, i}(x, y) y_{i}$ where $\ell_{n, i}(x, y) \in \mathbf{F}$ (otherwise, set $\ell_{n, i}=0$ ).

When $A$ is a field, we will denote linear disjointness over $A$ by $\downarrow_{A}^{\text {ld }}$.

One can easily check the following two facts.

Lemma 4.1. Let $M \models \mathrm{T}_{\mathrm{P}}$ and $A \subseteq M$. Then $A \leq M$ if and only if $A$ is a subring, $\mathbf{F}(A)$ is a field and $A \downarrow_{\mathbf{F}(A)}^{\text {ld }} \mathbf{F}(M)$.

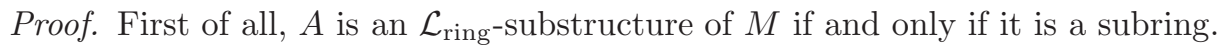

Let us now assume $A$ is closed under the functions $\ell_{n, i}$. Note that, if $x \in \mathbf{F}$, $\ell_{1,1}(1, x)=x^{-1}$ and hence $\mathbf{F}(A)$ is a field. Now, let $a \in A$ be a tuple. If $a$ is not linearly independent over $\mathbf{F}(M)$, we may assume that $a_{0}=\sum_{i>0} c_{i} a_{i}$ where the $\left(a_{i}\right)_{i>0}$ are linearly independent over $\mathbf{F}(M)$. Then $c_{i}=\ell_{n, i}(a) \in \mathbf{F}(A)$ and hence the tuple $a$ is not linearly independent over $\mathbf{F}(A)$. We have just proved that $A \downarrow_{\mathbf{F}(A)}^{\mathrm{ld}} \mathbf{F}(M)$.

Conversely, assume that $\mathbf{F}(A)$ is a field and $A \downarrow_{\mathbf{F}(A)}^{\text {ld }} \mathbf{F}(M)$. We have to show that $A$ is closed under the $\ell_{n, i}$ functions. Let $a$ be a tuple in $A$ such that $\left(a_{i}\right)_{i>0}$ is linearly independent over $\mathbf{F}(M)$ and $a_{0}=\sum_{i>0} \ell_{n, i}(a) a_{i}$. By hypothesis, the tuple $a$ is not linearly independent over $\mathbf{F}(A)$ either and (because $\mathbf{F}(A)$ is a field) there exist elements $c_{i} \in \mathbf{F}(A)$ such that $a_{0}=\sum_{i>0} c_{i} a_{i}$. Since the $\left(a_{i}\right)_{i>0}$ are linearly independent, we have $\ell_{n, i}(a)=c_{i} \in \mathbf{F}(A)$.

Lemma 4.2. Let $M_{i} \models \mathrm{T}_{\mathrm{P}}, A_{i} \leq M_{i}$ for $i=1,2, f: A_{1} \rightarrow A_{2}$ an $\mathcal{L}_{\text {ring- }}$ isomorphism such that $f\left(\mathbf{F}\left(A_{1}\right)\right)=\mathbf{F}\left(A_{2}\right)$. Then $f$ is in fact an $\mathcal{L}_{\mathrm{P}}$-isomorphism.

Proof. We have to check that $f$ respects the predicates $\mathbf{l d}_{n}$ and the functions $\ell_{n, i}$. First let $a \in A_{1}$ be a tuple. The tuple $a$ is linearly dependent over $\mathbf{F}\left(M_{1}\right)$ if and only if it is linearly dependent over $\mathbf{F}\left(A_{1}\right)$, i.e., there exist $\lambda_{i} \in \mathbf{F}\left(A_{1}\right)$ such that $\sum_{i} \lambda_{i} a_{i}=0$. Equivalently, $f\left(\sum_{i} \lambda_{i} a_{i}\right)=\sum_{i} f\left(\lambda_{i}\right) f\left(a_{i}\right)=0$ and the tuple $f(a)$ is linearly dependent over $\mathbf{F}\left(M_{2}\right)$. By symmetry, we also have that if $f(a)$ linearly dependent over $\mathbf{F}\left(M_{2}\right)$, then $a$ is linearly dependent over $\mathbf{F}\left(M_{1}\right)$. Hence $f$ respects $\mathbf{l d}_{n}$.

Let us now assume that $a$ is linearly independent over $\mathbf{F}\left(M_{1}\right)$ and that $c=$ $\sum_{i} \ell_{n, i}(c, a) a_{i}$. Then $f(c)=f\left(\sum_{i} \ell_{n, i}(c, a) a_{i}\right)=\sum_{i} f\left(\ell_{n, i}(c, a)\right) f\left(a_{i}\right)$. Moreover the tuple $(c, a)$ is linearly dependent over $\mathbf{F}\left(M_{1}\right)$ but $a$ is not and hence $(f(c), f(a))$ is linearly dependent over $\mathbf{F}\left(M_{2}\right)$ but $f(a)$ is not. Therefore $f(c)=$ $\sum_{i} \ell_{n, i}(f(c), f(a)) f\left(a_{i}\right)$ and by uniqueness of the coefficient in a decomposition of $f(c)$ in the basis $f(a)$, we obtain that $f\left(\ell_{n, i}(c, a)\right)=\ell_{n, i}(f(c), f(a))$. 
Lemma 4.3. Let $M_{i} \models \mathrm{T}_{\mathrm{P}}, A_{i} \leq M_{i}$ for $i=1,2, f: A_{1} \rightarrow A_{2}$ an $\mathcal{L}_{\mathrm{P}-}$ isomorphism. Then $\operatorname{Frac}\left(A_{i}\right) \leq M_{i}, \mathbf{F}\left(\operatorname{Frac}\left(A_{i}\right)\right)=\mathbf{F}\left(A_{i}\right)$ and $f$ extends to a unique $\mathcal{L}_{\mathrm{P}}$-isomorphism between $\operatorname{Frac}\left(A_{1}\right)$ and $\operatorname{Frac}\left(A_{2}\right)$.

Proof. One checks, by clearing the denominators, that $\operatorname{Frac}\left(A_{i}\right) \downarrow_{\mathbf{F}\left(A_{i}\right)}^{\text {ld }} \mathbf{F}\left(M_{i}\right)$ and thus that $\mathbf{F}\left(\operatorname{Frac}\left(A_{i}\right)\right)=\mathbf{F}\left(A_{i}\right)$. Lemmas 4.1 and 4.2 now allow us to conclude.

Lemma 4.4. Let $M_{i} \models \mathrm{T}_{\mathrm{P}}, A_{i} \leq M_{i}, \mathbf{F}\left(A_{i}\right) \leq_{\mathcal{L}_{\text {ring }}} C_{i} \leq_{\mathcal{L}_{\text {ring }}} \mathbf{F}\left(M_{i}\right)$ for $i=1,2$, $f: A_{1} \rightarrow A_{2}$ an $\mathcal{L}_{\mathrm{P}}$-isomorphism and $g: C_{1} \rightarrow C_{2}$ an $\mathcal{L}_{\text {ring-isomorphism such that }}$ $\left.g\right|_{\mathbf{F}\left(A_{1}\right)}=\left.f\right|_{\mathbf{F}\left(A_{1}\right)}$. Assume that $A_{i}$ and $C_{i}$ are fields then $A_{i} C_{i} \leq M_{i}, \mathbf{F}\left(A_{i} C_{i}\right)=$ $C_{i}$ and there is a unique $\mathcal{L}_{\mathrm{P}}$-isomorphism $h: A_{1} C_{1} \rightarrow A_{2} C_{2}$ extending $f$ and $g$.

Proof. As $A_{i} \downarrow_{\mathbf{F}\left(A_{i}\right)}^{\text {ld }} \mathbf{F}\left(M_{i}\right)$ and $C_{i} \leq \mathbf{F}\left(M_{i}\right)$, we have $A_{i} C_{i} \downarrow_{C_{i}}^{\text {ld }} \mathbf{F}\left(M_{i}\right)$. It follows that $\mathbf{F}\left(A_{i} C_{i}\right)=C_{i}$ and, by Lemma 4.1, that $A_{i} C_{i} \leq M_{i}$. As $A_{i} \downarrow_{\mathbf{F}\left(A_{i}\right)}^{\text {ld }} \mathbf{F}\left(M_{i}\right)$, it follows that $A_{i} C_{i}$ is isomorphic as a ring to $A_{i} \otimes_{\mathbf{F}\left(A_{i}\right)} C_{i}$ and hence there exists a unique ring isomorphism $h: A_{1} C_{1} \rightarrow A_{2} C_{2}$ extending $f$ and $g$. By Lemma 4.2, $h$ is in fact an $\mathcal{L}_{\mathrm{P}}$-isomorphism.

Lemma 4.5. Let $M_{i} \models \mathrm{T}_{\mathrm{P}}, A_{i} \leq M_{i}$ for $i=1,2$ and $f: A_{1} \rightarrow A_{2}$ an $\mathcal{L}_{\mathrm{P}-}$ isomorphism. Assume $A_{i}$ is a field and the extension $\mathbf{F}\left(A_{i}\right) \subseteq \mathbf{F}\left(M_{i}\right)$ is regular then $\overline{A_{i}} \cap M_{i} \leq M_{i}, \mathbf{F}\left(\overline{A_{i}} \cap M_{i}\right)=\mathbf{F}\left(A_{i}\right)$ and any field isomorphism $g: \overline{A_{1}} \cap M_{1} \rightarrow$ $\overline{A_{2}} \cap M_{2}$ extending $f$ is an $\mathcal{L}_{\mathrm{P}}$-isomorphism.

Proof. We have $A_{i} \downarrow_{\mathbf{F}\left(A_{i}\right)}^{\text {ld }} \mathbf{F}\left(M_{i}\right)$ and $\mathbf{F}\left(A_{i}\right) \subseteq \mathbf{F}\left(M_{i}\right)$ regular hence $A_{i} \subseteq A_{i} \mathbf{F}\left(M_{i}\right)$ is also regular, that is, $\overline{A_{i}} \downarrow_{A_{i}}^{\text {ld }} A_{i} \mathbf{F}\left(M_{i}\right)$. By transitivity of linear disjointness, we obtain that $\overline{A_{i}} \downarrow_{\mathbf{F}\left(A_{i}\right)}^{\text {ld }} \mathbf{F}\left(M_{i}\right)$ and hence $\overline{A_{i}} \cap M_{i} \downarrow_{\mathbf{F}\left(A_{i}\right)}^{\text {ld }} \mathbf{F}\left(M_{i}\right)$. In particular, we have $\mathbf{F}\left(\overline{A_{i}} \cap M_{i}\right)=\mathbf{F}\left(A_{i}\right)$ and we may conclude using Lemmas 4.1 and 4.2 .

Lemma 4.6. Let $M_{i} \models \mathrm{T}_{\mathrm{P}}, A_{i} \leq M_{i}, c_{i} \in M_{i}$ for $i=1,2$ and $f: A_{1} \rightarrow A_{2}$ an $\mathcal{L}_{\mathrm{P}}$-isomorphism. Assume $A_{i}$ is a field and $c_{i}$ transcendental over $A_{i} \mathbf{F}\left(M_{i}\right)$ then $A_{i}\left(c_{i}\right) \leq M_{i}, \mathbf{F}\left(A_{i}\left(c_{i}\right)\right)=\mathbf{F}\left(A_{i}\right)$ and there exists an $\mathcal{L}_{\mathrm{P}}$-isomorphism $g: A_{1}\left(c_{1}\right) \rightarrow$ $A_{2}\left(c_{2}\right)$ extending $f$ and sending $c_{1}$ to $c_{2}$.

Proof. We have that $f$ extends to a ring isomorphism $g$ on $A_{1}\left(c_{1}\right)$ sending $c_{1}$ to $c_{2}$. Moreover, $A_{i}\left(c_{i}\right)$ is algebraically independent from $A_{i} \mathbf{F}\left(M_{i}\right)$ over $A_{i}$. As $A_{i} \subseteq$ $A_{i}\left(c_{i}\right)$ is regular, $A_{i}\left(c_{i}\right) \downarrow_{A_{i}}^{\text {ld }} A_{i} \mathbf{F}\left(M_{i}\right)$. Since $A_{i} \downarrow_{\mathbf{F}\left(A_{i}\right)}^{\text {ld }} \mathbf{F}\left(M_{i}\right)$, by transitivity, it follows that $A_{i}\left(c_{i}\right) \downarrow_{\mathbf{F}\left(A_{i}\right)}^{\text {ld }} \mathbf{F}\left(M_{i}\right)$. In particular $\mathbf{F}\left(A_{i}\left(c_{i}\right)\right)=\mathbf{F}\left(A_{i}\right)$ and, by Lemma $4.2, g$ is in fact an $\mathcal{L}_{\mathrm{P}}$-isomorphism.

We can now prove a slightly improved version of $[5$, Theorem 1$]$. Let $\overline{T_{\mathrm{P}}}:=$ $\mathrm{T}_{\mathrm{P}} \cup \mathrm{ACF} \cup\{[\mathbf{K}: \mathbf{F}] \geq n \mid n \in \mathbb{N}\}$.

Theorem 4.7. The theory $\overline{T_{\mathrm{P}}}$ resplendently eliminates quantifiers relative to $\mathbf{F}$; that is, for every language $\widetilde{\mathcal{L}} \supseteq \mathcal{L}_{\text {ring }}$ and every $\widetilde{\mathcal{L}}$-theory $\widetilde{T}$ which eliminates quantifiers, the $\mathcal{L}_{\mathrm{P}} \cup \widetilde{\mathcal{L}}$-theory $\mathrm{T}_{\mathrm{P}}^{\star}:=\overline{\mathrm{T}_{\mathrm{P}}} \cup \widetilde{T}^{\mathbf{F}}$ eliminates quantifiers where $\widetilde{T}^{\mathbf{F}}$ is the relativization of $\widetilde{T}$ to $\mathbf{F}$.

Proof. Let us denote by $\mathcal{L}_{\mathrm{P}}^{\star}$ the language $\mathcal{L}_{\mathrm{P}} \cup \widetilde{\mathcal{L}}$. Note that an $\mathcal{L}_{\mathrm{P}}^{\star}$-isomorphism is an $\mathcal{L}_{\mathrm{P}}$-isomorphism that restricts to an $\widetilde{\mathcal{L}}$-isomorphism on $\mathbf{F}$. 
Let $M$ and $N$ be models of $\mathrm{T}_{\mathrm{P}}^{\star}, A \leq M$ and $f: A \rightarrow N$ an $\mathcal{L}_{\mathrm{P}}^{\star}$-embedding. Assume that $N$ is $|M|^{+}$-saturated. We have to show that $f$ extends to $M$. By Lemma 4.3, we may assume that $A$ is a field. Since $\mathbf{F}(\operatorname{Frac}(A))=\mathbf{F}(A)$, this new embedding is an $\widetilde{\mathcal{L}}$-embedding. By quantifier elimination in $\widetilde{T}$, we can extend $\left.f\right|_{\mathbf{F}(A)}$ to $g: \mathbf{F}(M) \rightarrow \mathbf{F}(N)$ and by Lemma 4.4, we may assume that $\mathbf{F}(M) \subseteq A$. As $\widetilde{T}$ eliminates quantifiers, $f(\mathbf{F}(M)) \preccurlyeq \widetilde{\mathcal{L}} \mathbf{F}(N)$ and this extension is regular. Applying Lemma 4.5, we may assume that $A$ is algebraically closed.

Claim 4.7.1. The transcendence degree of $N$ over $\mathbf{F}(N)$ is larger than $|M|$.

Proof. By compactness and saturation this follows from the fact that $N$ is an infinite extension of $\mathbf{F}(N)$.

Let $c \in M$ be transcendental over $A$ and $d \in N$ be transcendental over $f(A) \mathbf{F}(N)$. Then, by Lemma 4.6, $f$ extends to an $\mathcal{L}_{\mathrm{P}}$-embedding $g: A(c) \rightarrow N$ sending $c$ to $d$. Moreover, $\left.g\right|_{\mathbf{F}}=\left.f\right|_{\mathbf{F}}$ and hence $g$ is also an $\widetilde{\mathcal{L}}$-embedding. Now, by Lemma 4.5, $g$ extends to an $\mathcal{L}_{\mathrm{P}}^{\star}$-embedding on $\overline{A(c)}$. Repeating this last step sufficiently many times, we obtain an $\mathcal{L}_{\mathrm{P}}^{\star}$-embedding of $M$ into $N$.

4.1.2. The valued case. We now want to extend the previous results to the setting of dense pairs of valued fields.

Let $\mathcal{L}_{\mathrm{P}, \text { val }}:=\mathcal{L}_{\mathrm{P}} \cup \mathcal{L}_{\mathrm{RV}}$. We will consider the $\mathcal{L}_{\mathrm{P}, \text { val }}$-theory

$$
\mathrm{T}_{\mathrm{P}, \mathrm{val}}:=\mathrm{T}_{\mathrm{P}} \cup\{\mathbf{V F} \text { is a valued field and } \mathbf{F} \subseteq \mathbf{V F} \text { is dense }\} .
$$

In any model $M \models \mathrm{T}_{\mathrm{P} \text {,val }}$, by density of the pair $\mathbf{F}(M) \subseteq \mathbf{V F}(M)$, we have $\operatorname{rv}(\mathbf{V F}(M))=\operatorname{rv}(\mathbf{F}(M))$. We define $\mathbf{F}^{\mathbf{R V}}:=\mathbf{F} \cup \mathbf{R V}$.

Lemma 4.8. Let $M_{i} \models \mathrm{T}_{\mathrm{P}, \mathrm{val}}, A_{i} \leq M_{i}$ for $i=1,2$ and $f: A_{1} \rightarrow A_{2}$ an $\mathcal{L}_{\mathrm{P}, \text { val }}{ }^{-}$ isomorphism. Then $f$ extends to an $\mathcal{L}_{\mathrm{P}, \text { val }}$-isomorphism between $A_{1}\left(\operatorname{Frac}\left(\mathbf{V F}\left(A_{1}\right)\right)\right)$ and $A_{2}\left(\operatorname{Frac}\left(\mathbf{V F}\left(A_{2}\right)\right)\right)$.

Proof. By quantifier elimination in $\mathcal{L}_{\mathrm{RV}}, f$ extends to an $\mathcal{L}_{\mathrm{RV}}$-isomorphism $g$ : $A_{1}\left(\operatorname{Frac}\left(\mathbf{V F}\left(A_{1}\right)\right)\right) \rightarrow A_{2}\left(\operatorname{Frac}\left(\mathbf{V F}\left(A_{2}\right)\right)\right)$. By Lemma $4.3,\left.g\right|_{\mathbf{V F}}$ is an $\mathcal{L}_{\mathrm{P}}$-isomorphism.

Lemma 4.9. Let $M_{i} \models \mathrm{T}_{\mathrm{P}, \mathrm{val}}, A_{i} \leq M_{i}, \mathbf{F}^{\mathbf{R V}}\left(A_{i}\right) \leq_{\mathcal{L}_{\mathrm{RV}}} C_{i} \leq_{\mathcal{L}_{\mathrm{RV}}} \mathbf{F}^{\mathbf{R V}}\left(M_{i}\right)$ for $i=1,2, f: A_{1} \rightarrow A_{2}$ an $\mathcal{L}_{\mathrm{P}, \text { val }}$-isomorphism and $g: C_{1} \rightarrow C_{2}$ an $\mathcal{L}_{\mathrm{RV}}$-isomorphism such that $\left.g\right|_{\mathbf{F}^{\mathrm{RV}}\left(A_{1}\right)}=\left.f\right|_{\mathbf{F}^{\mathrm{RV}}\left(A_{1}\right)}$. Moreover, assume that for all $a \in \mathbf{V F}\left(A_{i}\right) \backslash$ $\mathbf{V F}\left(C_{i}\right)$ there is a Cauchy sequence $\left(c_{\alpha}^{a}\right)$ in $\mathbf{V F}\left(C_{i}\right)$ converging to a (in $\mathbf{V F}\left(A_{i}\left(C_{i}\right)\right)$ ) and such that $g\left(c_{\alpha}^{a}\right)=c_{\alpha}^{f(a)}$. Assume that $\mathbf{V F}\left(A_{i}\right)$ and $\mathbf{V F}\left(C_{i}\right)$ are fields. Then

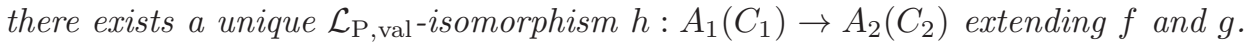

Proof. Our assumptions imply that $\mathbf{V F}\left(C_{i}\right)$ is dense in $\mathbf{V F}\left(A_{i}\left(C_{i}\right)\right)$. By Lemma 4.4, there exists a unique $\mathcal{L}_{\mathrm{P}}$-isomorphism $h: \mathbf{V F}\left(A_{1}\left(C_{1}\right)\right) \rightarrow \mathbf{V F}\left(A_{2}\left(C_{2}\right)\right)$ extending $\left.f\right|_{\mathbf{V F}}$ and $\left.g\right|_{\mathbf{V F}}$. We extend it to $\mathbf{R V}$ by setting $\left.h\right|_{\mathbf{R V}}:=\left.g\right|_{\mathbf{R V}}$. Note that, by density, $\mathbf{R V}\left(A_{i}\left(C_{i}\right)\right)=\mathbf{R V}\left(C_{i}\right)$.

We have to check that $h$ preserves rv. Let us assume that all $\left(c_{\alpha}^{a}\right)$ are indexed by the same ordinal (the cofinality of $\operatorname{val}\left(\mathbf{V F}\left(C_{i}\right)^{\star}\right)$ ). Let $P(\bar{X}) \in C_{1}[\bar{X}]$ and $a \in$ $\mathbf{V F}\left(A_{1}\right)^{|\bar{X}|}$. If $P(a)=0$ then $h(P(a))=0$ and $\operatorname{rv}(h(P(a)))=\infty=h(\operatorname{rv}(P(a)))$. Thus, we may assume that $P(a) \neq 0$ and $P^{g}(f(a)) \neq 0$. Since val $\left(a-c_{\alpha}^{a}\right)$ is cofinal in $\operatorname{val}\left(\mathbf{V F}\left(A_{1}\left(C_{1}\right)\right)\right)$ and $P\left(c_{\alpha}^{a}\right)=P(a)+\sum_{I \neq 0}\left(c_{\alpha}^{a}-a\right)^{I} P_{I}(a)$, for large enough $\alpha$, one has $\operatorname{rv}(P(a))=\operatorname{rv}\left(P\left(c_{\alpha}^{a}\right)\right)$. As $g$ is an $\mathcal{L}_{\mathrm{RV}}$-isomorphism, $g\left(\operatorname{rv}\left(P\left(c_{\alpha}^{a}\right)\right)\right)=$ 
$\operatorname{rv}\left(P^{g}\left(c_{\alpha}^{f(a)}\right)\right)$. Similarly, $\operatorname{rv}\left(P^{g}\left(c_{\alpha}^{f(a)}\right)\right)=\operatorname{rv}\left(P^{g}(f(a))\right)=\operatorname{rv}(h(P(a)))$. Hence $h(\operatorname{rv}(P(a)))=g\left(\operatorname{rv}\left(P\left(c_{\alpha}^{a}\right)\right)\right)=\operatorname{rv}(h(P(a)))$.

Corollary 4.10. Let $M_{i} \models \mathrm{T}_{\mathrm{P}, \mathrm{val}}, A_{i} \leq M_{i}, \mathbf{F}^{\mathbf{R V}}\left(A_{i}\right) \leq{\mathcal{L}_{\mathrm{RV}}}_{i} C_{\mathcal{L}_{\mathrm{RV}}} \mathbf{F}^{\mathbf{R V}}\left(M_{i}\right)$ for $i=1,2, f: A_{1} \rightarrow A_{2}$ an $\mathcal{L}_{\mathrm{P}, \mathrm{val}}$-isomorphism and $g: C_{1} \rightarrow C_{2}$ an $\mathcal{L}_{\mathrm{RV}}$-isomorphism such that $\left.g\right|_{\mathbf{F}^{\mathrm{RV}}\left(A_{1}\right)}=\left.f\right|_{\mathbf{F}^{\mathrm{RV}}\left(A_{1}\right)}$.

Assume that $\mathbf{F}\left(A_{i}\right)$ is dense in $\mathbf{V F}\left(A_{i}\right), \operatorname{val}\left(\mathbf{V F}\left(A_{i}\right)^{\star}\right)$ is a cofinal subset of $\operatorname{val}\left(\mathbf{V F}\left(A_{i}\left(C_{i}\right)\right)^{\star}\right)$ and that $\mathbf{V F}\left(A_{i}\right)$ and $\mathbf{V F}\left(C_{i}\right)$ are fields. Then there exists a unique $\mathcal{L}_{\mathrm{P}, \mathrm{val}}$-isomorphism $h: A_{1}\left(C_{1}\right) \rightarrow A_{2}\left(C_{2}\right)$ extending $f$ and $g$.

Proof. This is an immediate consequence of Lemma 4.9. Indeed, for all $a \in A_{1} \backslash C_{1}$, let $c_{\alpha}^{a}$ be Cauchy sequence in $\mathbf{F}\left(A_{1}\right)$ converging to $a$ in $\mathbf{V F}\left(A_{1}\right)$ (and hence in $\mathbf{V F}\left(A_{1}\left(C_{1}\right)\right)$ by cofinality) and define $c_{\alpha}^{f(a)}$ to be equal to $f\left(c_{\alpha}^{a}\right)$. Now, Lemma 4.9 applies.

Let $\mathcal{L}$ be an $\mathbf{R V}$-enrichment of $\mathcal{L}_{\mathrm{RV}}$ and $T$ be an $\mathcal{L}$-theory of valued fields that eliminates quantifiers. The main two examples are ACVF and theories of equicharacteristic zero Henselian fields Morleyized on RV. As always, the present results remain true in mixed characteristic provided $\mathcal{L}_{\mathrm{RV}}$ is understood as the language of higher order leading terms, i.e., with sorts for $\mathbf{R V}_{n}:=\mathbf{V F} /(1+n \mathfrak{m})$ for all $n \in \mathbb{N}$. Let $\widetilde{\mathcal{L}} \supseteq \mathcal{L}$ be a language and $\widetilde{T}$ be an $\widetilde{\mathcal{L}}$-theory which eliminates quantifiers. We will now consider the theory

$$
\mathrm{T}_{\mathrm{P}, \mathrm{val}}^{\star}:=\mathrm{T}_{\mathrm{P}, \mathrm{val}} \cup T \cup \widetilde{T}^{\mathbf{F}^{\mathrm{RV}}} \cup\{[\mathbf{V F}: \mathbf{F}] \geq n \mid n \in \mathbb{N}\} .
$$

We will be denoting by $\mathbf{B}$ the (interpretable) set of open balls.

Theorem 4.11. Assume that:

(1) For all $M \models \mathrm{T}_{\mathrm{P}, \text { val }}^{\star}, A \leq \mathbf{F}(M)$ and tuples $b_{1}, b_{2} \in \mathbf{B}(M)$ : If $b_{1} \equiv_{\mathcal{L}(A)}^{M} b_{2}$ then $b_{1} \equiv \underset{\widetilde{\mathcal{L}}(A)}{\mathbf{F}(M)} b_{2}$.

(2) For every $A \leq \mathbf{F}(M) \models \widetilde{T}$ with $M$ sufficiently saturated with respect to $|A|$, $C_{A}:=\{x \in \mathbf{V F}(M) \mid$ the $\widetilde{\mathcal{L}}$-structure and the $\mathcal{L}$-structure generated by $A x$ are equal\} is dense in $\mathbf{V F}(M)$.

Then $\mathrm{T}_{\mathrm{P}, \mathrm{val}}^{\star}$ eliminates quantifiers. In particular, if $M \models \mathrm{T}_{\mathrm{P}, \mathrm{val}}^{\star}$, then $\mathbf{F}(M)$ is stably embedded in $M$ with induced structure given by $\widetilde{\mathcal{L}}$.

Proof. Let $M$ and $N$ be models of $\mathrm{T}_{\mathrm{P}, \text { val }}^{\star}, A \leq M$ and $f: A \rightarrow N$ an $\mathcal{L}_{\mathrm{P}, \mathrm{val}^{-}}^{\star}$ embedding, where $\mathcal{L}_{\mathrm{P}, \text { val }}^{\star}:=\mathcal{L}_{\mathrm{P}, \text { val }} \cup \widetilde{\mathcal{L}}$. Assume that $N$ is $|M|^{+}$-saturated. We have to show that $f$ extends to $M$. By Lemma 4.8, we may assume that $\operatorname{VF}(A)$ is a field.

Claim 4.11.1. At the cost of enlarging $M$ (without changing its cardinality) and $A \leq M$, we may assume that $\mathbf{F}(A)$ is dense in $\mathbf{V F}(A)$ and $\operatorname{val}\left(\mathbf{V F}(A)^{\star}\right)$ is cofinal in $\operatorname{val}\left(M^{\star}\right)$.

Proof. Let $M^{\prime}$ be some sufficiently saturated extension of $M$. Let $\kappa:=|\mathbf{V F}(A)|$ and $\left(a_{i}\right)_{i \in \kappa}$ be an enumeration of $\mathbf{V F}(A)$.

By induction on $(n, i) \in \omega \times \kappa$ (ordered lexicographically), we will construct

- a sequence $\left(e_{n, i}\right)_{n \in \omega, i \in \kappa}$ in $\mathbf{F}\left(M^{\prime}\right)$;

- an increasing chain $\left(M_{n, i}\right)_{n \in \omega, i \in \kappa}$ of elementary substructures of $M^{\prime}$ containing $M$; 
- an increasing chain of $\mathcal{L}_{\mathrm{P}, \mathrm{val}}^{\star}$-embeddings $f_{n, i}: A_{n, i} \rightarrow N$ extending $f$, where $A_{n, i}$ is the structure generated by $A \cup\left\{e_{m, j} \mid(m, j) \leq(n, i)\right\}$,

satisfying the following conditions for all $(n, i) \in \omega \times \kappa$ :

(i) $\left|M_{n, i}\right|=|M|$;

(ii) $e_{n, i} \in M_{n, i}$;

(iii) $\operatorname{val}\left(e_{n, i}-a_{i}\right)>\operatorname{val}\left(M_{<(n, i)}^{\star}\right)$, where $M_{<(n, i)}:=M \cup \bigcup_{\left(n^{\prime}, i^{\prime}\right)<(n, i)} M_{n^{\prime}, i^{\prime}}$.

We will denote the image of $f$ by $C$, and that of $f_{n, i}$ by $C_{n, i}$.

Let us first construct $e_{0,0}, M_{0,0}$ and $f_{0,0}$. By saturation of $M^{\prime}$, and Hypothesis 2 , we find $e_{0,0} \in \mathbf{F}\left(M^{\prime}\right)$ such that $\operatorname{val}\left(e_{0,0}-a_{0}\right)>\boldsymbol{\Gamma}(M) \backslash\{\infty\}$ and the $\widetilde{\mathcal{L}}$-structure and the $\mathcal{L}$-structure generated by $\mathbf{F}(A) e_{0,0}$ are equal. We also find an elementary submodel $M_{0,0}$ of $M^{\prime}$ of cardinality $|M|$ containing $M\left(e_{0,0}\right)$. Let us now show that we may extend $f$ to $e_{0,0}$.

Note that Hypothesis 1 implies that if $\eta: A \rightarrow N$ is an $\widetilde{\mathcal{L}}$-isomorphism between subsets of $\mathbf{F}$ and $\rho$ is an extension of $\eta$ to some tuple of balls $b$, which is an $\mathcal{L}$ embedding, then there exists an $\widetilde{\mathcal{L}}$-embedding extending $\eta$ and sending $b$ to $\rho(b)$. Indeed, by quantifier elimination in $\widetilde{\mathcal{L}}, \eta^{-1}$ can be extended to an $\widetilde{\mathcal{L}}$-embedding $i$ defined at $\rho(b)$. Then $i \circ \rho$ fixes $A$. So applying Hypothesis 1 to $b$ and $i(\rho(b))$, we get that there exists an $\widetilde{\mathcal{L}}$-embedding $j$ fixing $A$ and sending $b$ to $i(\rho(b))$. Then $h:=i^{-1} \circ j$ is an $\widetilde{\mathcal{L}}$-embedding extending $\eta$ and sending $b$ to $\rho(b)$.

Let us now come back to our previous notations. By quantifier elimination in $T$, $f$ extends to an elementary $\mathcal{L}$-embedding $g: M_{0,0} \rightarrow N$. By the previous paragraph and quantifier elimination in $\widetilde{T}$, there exists an $\widetilde{\mathcal{L}}$-embedding $h: \mathbf{F}\left(M_{0,0}\right) \rightarrow \mathbf{F}(N)$ extending $\left.f\right|_{\mathbf{F}}$ such that $\left.h\right|_{\mathbf{B}\left(M_{0,0}\right)}=\left.g\right|_{\mathbf{B}\left(M_{0,0}\right)}$. Let $c_{0,0}=h\left(e_{0,0}\right) \in \mathbf{F}(N)$. By construction, we have that $\operatorname{tp}_{\widetilde{\mathcal{L}}}\left(c_{0,0} / \mathbf{F}(C)\right)=f_{\star} \operatorname{tp}_{\widetilde{\mathcal{L}}}\left(e_{0,0} / \mathbf{F}(A)\right)$.

Let $d \in M, \gamma:=\operatorname{rv}\left(e_{0,0}-d\right)$ and $b:=\{x \mid \operatorname{rv}(x-d)=\gamma\}$. We have $e_{0,0} \in$ $b \in \mathbf{B}\left(M_{0,0}\right)$ and hence $c_{0,0} \in h(b)=g(b)=\{x \mid \operatorname{rv}(x-g(d))=g(\gamma)\}$. It follows that $\operatorname{rv}\left(c_{0,0}-g(d)\right)=g\left(\operatorname{rv}\left(e_{0,0}-d\right)\right)$. In particular val $\left(c_{0,0}-f\left(a_{0}\right)\right)>\boldsymbol{\Gamma}(M) \backslash\{\infty\}$ and hence for all non-zero polynomials $P=\sum_{i} g\left(d_{i}\right) X^{i} \in \mathbf{V F}(g(M))[X]$, letting $i_{0}=\min \left\{i \mid g\left(d_{i}\right) \neq 0\right\}$, we have

$$
\begin{aligned}
\operatorname{rv}\left(P\left(c_{0,0}-f\left(a_{0}\right)\right)\right)=\operatorname{rv}\left(g\left(d_{i_{0}}\right)\right) & \cdot \operatorname{rv}\left(c_{0,0}-f\left(a_{0}\right)\right)^{i_{0}} \\
& =g\left(\operatorname{rv}\left(d_{i_{0}}\left(e_{0,0}-a_{0}\right)^{i_{0}}\right)=g\left(\operatorname{rv}\left(P\left(e_{0,0}-a_{0}\right)\right)\right)\right.
\end{aligned}
$$

It follows (since $g$ is an $\mathcal{L}$-isomorphism) that $\operatorname{tp}_{\mathcal{L}}\left(c_{0,0} / g(M)\right)=g_{\star} \operatorname{tp}_{\mathcal{L}}\left(e_{0,0} / M\right)$. In particular, $\operatorname{tp}_{\mathcal{L}}\left(c_{0,0} / C\right)=f_{\star} \operatorname{tp}_{\mathcal{L}}\left(e_{0,0} / A\right)$.

Let $f_{0,0}$ be an $\mathcal{L}$-embedding extending $f$ to $A\left(e_{0,0}\right)$ and sending $e_{0,0}$ to $c_{0,0}$. Then $\left.f_{0,0}\right|_{\mathrm{F}}$ is an $\widetilde{\mathcal{L}}$-embedding (remember that the $\widetilde{\mathcal{L}}$-structure and the $\mathcal{L}$-structure generated by $\mathbf{F}(A) e_{0,0}$ are equal). Finally, by Lemma $4.4, f_{0,0}$ is also an $\mathcal{L}_{\mathrm{P}}$-embedding.

Now let $(n, i)>(0,0)$ be given, and assume that $e_{m, j}, M_{m, j}$ as well as $f_{m, j}$ have been constructed for all $(m, j)<(n, i)$ satisfying the above. We may then construct $e_{n, i}, M_{n, i}$ and $f_{n, i}$ in the exact same way. In the argument above, it is enough to replace $M$ by $M_{<(n, i)}, A$ by $A_{<(n, i)}=\bigcup_{(m, j)<(n, i)} A_{m, j}$ and $f$ by $f_{<(n, i)}=\bigcup_{(m, j)<(n, i)} f_{m, j}$.

We define $f_{<(\omega, \kappa)}, M_{<(\omega, \kappa)}$ and $A_{<(\omega, \kappa)} \leq M_{<(\omega, \kappa)}$ similarly. It is easy to check that $\operatorname{val}\left(\mathbf{F}\left(A_{<(\omega, \kappa)}^{\star}\right)\right)$ is cofinal in $\operatorname{val}\left(M_{<(\omega, \kappa)}^{\star}\right)$ and, as the $\left(e_{n, i}\right)_{n \in \omega}$ are Cauchy sequences whose limit is $a_{i}$ in $\mathbf{V F}\left(A_{<(\omega, \kappa)}\right)$, that $\mathbf{F}\left(A_{<(\omega, \kappa)}\right)$ is dense in $\mathbf{V F}\left(A_{<(\omega, \kappa)}\right)$. 
We can now apply Corollary 4.10 to extend $f$ to all of $\mathbf{F}(M)$ and we may assume that $\mathbf{F}(A)=\mathbf{F}(M)$. We may now extend $f$ to the relative algebraic closure of $A$ in $M$ using Lemma 4.5 and quantifier elimination in $T$.

Claim 4.11.2. Any ball from $N$ has transcendence degree larger or equal to $|M|^{+}$ over $\mathbf{F}(N)$.

Proof. It suffices to prove the claim for $\mathcal{O}$ and, in that case, it is an easy consequence of Claim 4.7.1.

Now let $a \in M \backslash A$, then $a \in \mathbf{V F}(M)$, and $a$ is transcendental over $\mathbf{V F}(A)$. Let $\left(a_{\alpha}\right)$ be a Cauchy-sequence in $A$ converging to $a$. Note that val $\left(a-a_{\alpha}\right)$ is cofinal in $\Gamma(M)$. Let $b$ be a ball in $N$ that only contains pseudo-limits of the sequence $\left(f\left(a_{\alpha}\right)\right)$. By Claim 4.11.2, we can find a point $c \in b$ which is transcendental over $\mathbf{V F}(f(A)) \mathbf{F}(N)$. Let $P \in \mathbf{V F}(A)[X]$, then $v(P(a))<\infty$ and for large enough $\alpha, \operatorname{rv}(P(a))=\operatorname{rv}\left(P\left(a_{\alpha}\right)\right)$ (cf. the proof of Lemma 4.9). Similarly, $\operatorname{rv}\left(P^{f}(c)\right)=$ $\operatorname{rv}\left(P^{f}\left(f\left(a_{\alpha}\right)\right)\right)=f(\operatorname{rv}(P(a)))$. It follows that $f$ can be extended to an $\mathcal{L}$-embedding sending $a$ to $c$. By Lemma 4.6, this extension is an $\mathcal{L}_{\mathrm{P}, \mathrm{val}}^{\star}$-embedding. Repeating these last two steps we can extend $f$ to $M$.

Remark 4.12. The hypotheses of Theorem 4.11 are verified in the two following cases:

- If $\widetilde{T}=T$, Hypothesis 1 follows immediately from the fact that $\widetilde{\mathcal{L}}=\mathcal{L}$ and the fact that $\mathbf{F}(M) \preccurlyeq \mathcal{L} M$. Hypothesis 2 is trivial in this case as $\widetilde{\mathcal{L}}=\mathcal{L}$. The previous result therefore applies to dense elementary extensions of characteristic zero Henselian fields.

- Let $T=\operatorname{ACVF}_{p, p}$ and $\widetilde{T}=\mathrm{SCVH}_{p, e}$ (or $\mathrm{SCVF}_{p, e}$, respectively). Hypothesis 1 follows from the fact that if $b_{1} \equiv_{\mathcal{L}_{\mathrm{RV}}(A)}^{M} b_{2}$ for $b_{1}, b_{2} \in \mathbf{B}(M)=$ $\mathbf{B}(\mathbf{F}(M))$ and $A \leq \mathbf{F}(M)$ then $b_{1} \equiv_{\mathcal{L}_{\mathcal{G}}(A)}^{M} b_{2}$ (since the additional sorts in $\mathcal{L}_{\mathcal{G}}$ are interpretable) and thus $b_{1} \equiv_{\mathcal{L}_{\mathcal{G}, p, e}^{D}(A)}^{\mathbf{F}(M)} b_{2}$ (similarly for $\mathcal{L}_{\mathcal{G}, p, e}^{\lambda}$ ) by Corollary 2.18.

Hypothesis 2 follows from the fact that for all $A, \mathbf{F}(M)_{p \infty} \subseteq C_{A}$ and $\mathbf{F}(M)_{p^{\infty}}$ is dense in $\mathbf{F}(M)$, a field which is dense in $\mathbf{V F}(M)$ by assumption.

4.2. Dense pairs (ACVF, SCVF). We will now focus on the case $T=\operatorname{ACVF}_{p, p}^{\mathcal{G}}$ and $\widetilde{T}=\operatorname{SCVH}_{p, e}^{\mathcal{G}}$ (there are similar corollaries in the case $\widetilde{T}=\operatorname{SCVF}_{p, e}^{\mathcal{G}}$ ). Note that the geometric language does not exactly fit in the setting of the previous section (since there are additional geometric sorts), but for the results that we are giving here the precise language in which we are working is completely immaterial.

Corollary 4.13. In models of $\mathrm{T}_{\mathrm{P}, \mathrm{val}}^{\star}, \boldsymbol{\Gamma}$ is stably embedded and a pure divisible ordered Abelian group and $\mathbf{k}$ is stably embedded and a pure algebraically closed field.

Proof. It follows from Theorem 4.11, that RV is stably embedded in models of $\mathrm{T}_{\mathrm{P} \text {,val }}^{\star}$ and that its structure is the structure induced by ACVF. Stable embeddedness and purity of $\boldsymbol{\Gamma}$ and $\mathbf{k}$ now follow from the equivalent result in ACVF.

Corollary 4.14. The theory $\mathrm{T}_{\mathrm{P}, \mathrm{val}}^{\star}$ is complete.

Proof. The field $\mathbb{F}_{p}$ can be embedded, as a subset of $\mathbf{F}$, in every model of $\mathrm{T}_{\mathrm{P}, \text { val }}^{\star}$. Completeness follows from Theorem 4.11. 
Lemma 4.15. Let $K \models \mathrm{SCVF}$. The following are equivalent:

(i) $K$ does not admit any separable immediate valued field extension, i.e., $K$ is separably maximally complete;

(ii) $\bar{K}$ does not admit any immediate valued field extension, i.e., $\bar{K}$ is maximally complete.

Proof. Let us first prove that (i) implies (ii). Let $\bar{K} \subseteq L$ be a proper immediate extension. We may assume that $L=\bar{K}(t)$, where $t$ is a transcendental element over $\bar{K}$. But then $K(t) / K$ is immediate (and separable of course).

Conversely, assume that $K$ has a proper immediate separable extension. As $K$ is separably closed, this cannot be an algebraic extension and we may thus assume it is of the form $K(t)$ with $t$ transcendental. As $\boldsymbol{\Gamma}(K)=\boldsymbol{\Gamma}(K(t))$ is divisible and $\mathbf{k}(K)=\mathbf{k}(K(t))$ is algebraically closed, the algebraic extension $\bar{K}(t) / K(t)$ is immediate, and so $\bar{K}(t)$ is a proper immediate extension of $\bar{K}$.

Proposition 4.16. The theory $\mathrm{T}_{\mathrm{P}, \mathrm{val}}^{\star}$ admits a model $M$ such that $\boldsymbol{\Gamma}(M)=\mathbb{R}$ and $\mathbf{V F}(M)$ is maximally complete.

Proof. Let $\left(x_{\alpha}\right)_{\alpha \in 2^{\aleph_{0}}}$ be a linear basis of $\mathbb{R}$ over $\mathbb{Q}$. Let $L_{0}:=\mathbb{F}_{p}\left(t_{i}\right)_{1 \leq i \leq e}$ be trivially valued and $L_{1}:=L_{0}\left(x_{\alpha}^{p^{-\infty}}: \alpha \in 2^{\aleph_{0}}\right)$ be equipped with the unique valuation such that $\operatorname{val}\left(x_{\alpha}\right)=x_{\alpha}$. Then $L_{2}:=\bar{L}_{1}$ sep is separably closed of characteristic $p$ and Ershov invariant $e$ and so is any separably maximally complete extension $L \supseteq L_{1}$. The pair $(\bar{L}, L)$ is dense and $\operatorname{val}(\bar{L})=\mathbb{Q} \otimes\left\langle x_{\alpha}\right\rangle_{\alpha \in 2^{\aleph_{0}}}=\mathbb{R}$. Then $L$ can be endowed with Hasse derivations so that $(\bar{L}, L) \models \mathrm{T}_{\mathrm{P}, \text { val }}^{\star}$.

Moreover, by Lemma $4.15, \bar{L}$ is maximally complete.

4.3. Imaginaries in $\mathrm{SCVH}_{p, e}$. We begin with a review of some results from [23, 24]. Consider an arbitrary complete theory $T$, and a fixed universal domain M. As usual, for a definable set $\mathbf{X}$ over parameters, we denote by $\operatorname{acl}(\ulcorner\mathbf{X}\urcorner)$ the set of elements of $M$ whose orbit under the (set-wise) stabilizer of $\mathbf{X}(\mathrm{M})$ in $\operatorname{Aut}(\mathrm{M})$ is finite. We will use the following criterion for elimination of imaginaries:

Proposition 4.17 ([23, Proposition 10.1]). Assume that every non-empty definable set $\mathbf{X}$ in a theory $T$ contains an $\operatorname{acl}(\ulcorner\mathbf{X}\urcorner)$-invariant global type. Then $T$ admits weak elimination of imaginaries.

In fact, it suffices to consider sets $\mathbf{X}$ in (powers of) a dominant sort.

While the above criterion holds with invariant types, we will actually show the existence of a definable type in $\mathbf{X}$. This is shown in several steps. The following result from [23] produces an ACVF-type consistent with a given definable set in a suitable expansion, definable in that expansion:

Proposition 4.18 ([23, Proposition 9.6]). Let $\widehat{T} \supseteq \mathrm{ACVF}^{\mathcal{G}}$ be a theory in a countable language $\widehat{\mathcal{L}}$, such that

(1) $\widehat{T}$ eliminates imaginaries;

(2) $\mathbf{k}$ and $\boldsymbol{\Gamma}$ are stably embedded, and

(3) The induced theories on $\mathbf{k}$ and $\boldsymbol{\Gamma}^{\mathrm{eq}}$ eliminate $\exists^{\infty}$.

Let $\widehat{A}=\operatorname{acl}_{\widehat{T}}(\widehat{A}) \subseteq \widehat{N} \models \widehat{T}$ and let $\mathbf{X}$ be a non-empty strict pro- $\widehat{A}$-definable set of $\mathbf{V F}$ elements. Then there exists a global $\mathrm{ACVF}^{\mathcal{G}}$-type $p$ consistent with $\mathbf{X}$, which is $\widehat{A}$-definable (in $\widehat{T}$ ). 
To replace definability in $\widehat{T}$ with definability in $\mathrm{ACVF}^{\mathcal{G}}$, we will use the following result. We recall that a subset $A$ of a structure $\mathrm{M}$ is uniformly stably embedded if for every formula $\varphi(x, y)$ there is a formula $\psi(x, z)$ such that for every $m \in$ M there is $a \in A$ such that $\varphi(A, m)=\psi(A, a)$. We have:

Proposition 4.19 ([24, Corollary 1.7]). Let $T$ be an NIP $\mathcal{L}$-theory that eliminates imaginaries. Let $\widehat{T} \supseteq T$ be a complete $\widehat{\mathcal{L}}$-theory that also eliminates imaginaries. Suppose that there exists $\widehat{M} \models \widehat{T}$ such that $\left.\widehat{M}\right|_{\mathcal{L}}$ is uniformly stably embedded in every elementary extension. Let $\widehat{N} \models \widehat{T}, \widehat{A}=\operatorname{dcl}_{\widehat{T}}(\widehat{A}) \subseteq \widehat{N}$ and $p$ be a global $\mathcal{L}$-type. If $p$ is $\widehat{A}$-definable in $\widehat{T}$, then it is in fact $\left.\widehat{A}\right|_{\mathcal{L}}$-definable in $T$.

We now go back to our context. We set $T=\operatorname{ACVF}_{p, p}^{\mathcal{G}}$ and $\widehat{T}=\left(\mathrm{T}_{\mathrm{P}, \mathrm{val}}^{\star}\right)^{\mathrm{eq}}$. Combining the last two results, we obtain:

Corollary 4.20. Let $\mathbf{X}$ be a non-empty strict pro-definable set of $\mathbf{V F}$ elements in

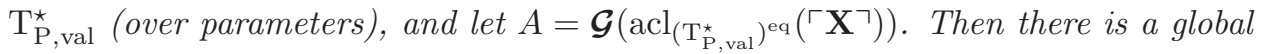
$\mathrm{ACVF}_{p, p}^{\mathcal{G}}$-type $p, A$-definable in $\mathrm{ACVF}_{p, p}^{\mathcal{G}}$ and consistent with $\mathbf{X}$.

Proof. Let $\widehat{A}$ be the algebraic closure of the code of $\mathbf{X}$ in $\widehat{T}=\left(\mathrm{T}_{\mathrm{P}, \text { val }}^{\star}\right)^{\text {eq }}$. Corollary 4.13 shows that the hypothesis of Proposition 4.18 holds, and therefore provides us with an $\widehat{A}$-definable (in $\widehat{T}$ ) global $T$-type $p$, consistent with $\mathbf{X}$. Now, [23, Corollary A.7] asserts that the model provided by Proposition 4.16 satisfies the condition in Proposition 4.19, and Corollary 4.14 shows that $\mathrm{T}_{\mathrm{P}, \mathrm{val}}^{\star}$ (hence $\widehat{T}$ ) is complete, so Proposition 4.19 applies to show that $p$ is definable in $T$ over $\mathcal{G}(\widehat{A})=A$.

Recall that if $a$ is an element of a model of $\operatorname{SCVH}_{p, e}$, we denote by $D_{\omega}(a)$ the infinite tuple obtained by applying the derivations to $a$. If $p(x)$ is a (partial) type in the field sort of $\mathrm{SCVH}_{p, e}$, we let $\nabla(p)$ be the pro-definable set in the language of ACVF determined by the condition: $D_{\omega}(a) \models \nabla(p)$ if and only if $a \models p$ for all tuples $a$ in a model of $\mathrm{SCVH}_{p, e}$ (in other words, $\nabla(p)$ is the prolongation of $p$ ).

If $\mathbf{X}$ is a definable set in $\mathrm{SCVH}_{p, e}$ (in some $\mathbf{V} \mathbf{F}^{n}$ ), then its image $D_{\omega}(\mathbf{X})$ is a strict pro-definable set over the same parameters, and for all types $p$ of $\mathrm{SCVH}_{p, e}, p$ is consistent with $\mathbf{X}$ if and only if $\nabla(p)$ is consistent with $D_{\omega}(\mathbf{X})$. Furthermore, any complete $\mathrm{ACVF}_{p, p}$ type consistent with $D_{\omega}(\mathbf{X})$ is of the form $\nabla(p)$ for a complete $\mathrm{SCVF}_{p, e}$ type in $\mathbf{X}$, over the same parameters. Thus, $\nabla$ provides a bijection between

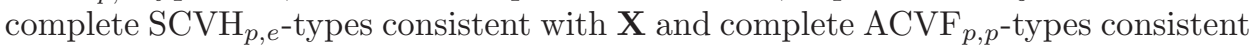
with $D_{\omega}(\mathbf{X})$, over the same parameters.

Theorem 4.21. Let $\widetilde{N} \models \mathrm{SCVH}_{p, e}^{\mathcal{G}}$ and $\mathbf{X} \subseteq \mathbf{V F}^{n}$ be $\widetilde{N}$-definable. Let $A=$ $\operatorname{acl}(\ulcorner\mathbf{X}\urcorner)$. Then, there exists an A-definable type $p$ such that $p(x) \vdash x \in \mathbf{X}$.

Proof. Let us assume that $\widetilde{N}$ is sufficiently saturated and let $N$ denote its algebraic closure. Then $N_{P}:=(N, \widetilde{N}) \models \mathrm{T}_{\mathrm{P} \text {,val }}^{\star}$. Replacing $\mathbf{X}$ with $D_{\omega}(\mathbf{X})$ as above, it suffices to find an $A$-definable $\mathrm{ACVF}_{p, p}$-type $p$.

Let $B=\operatorname{acl}_{\mathcal{L}_{\mathrm{P}, \text { val }}^{\star}}^{\text {eq }}(\ulcorner\mathbf{X}\urcorner)$. According to Corollary $4.20, \mathbf{X}$ contains an $\operatorname{ACVF}_{p, p^{-}}^{\mathcal{G}}$ type $p$ definable over $\mathcal{G}(B)$. To complete the proof, it remains to show that $\mathcal{G}(B)$ is contained in $\operatorname{dcl}_{N}(A)$. To establish this, by Lemma 3.8(2) it is enough to show that $\mathcal{G}(B) \cap \widetilde{N} \subseteq A$. This latter inclusion follows from the fact that $\widetilde{N}$ is stably embedded in $N_{P}$ as a pure model of $\mathrm{SCVH}_{p, e}^{\mathcal{G}}$ (by Theorem 4.11). 
Let again $T$ be an arbitrary theory. In case $T$ eliminates imaginaries, the condition in Prop. 4.17 can be viewed as asserting the density of invariant types in the space of types over acl $(\ulcorner X\urcorner)$. Applying compactness, one obtains:

Proposition 4.22 ([23, Proposition 10.2]). For a theory $T=T^{\mathrm{eq}}$ and a set of parameters $A$, the following are equivalent:

(1) Every A-definable set contains an A-invariant type.

(2) (the invariant extension property over A) Every type over $A$ extends to a global A-invariant type.

Combining everything, we obtain:

Corollary 4.23. The theory $\mathrm{SCVH}_{p, e}^{\mathcal{G}}$ eliminates imaginaries and has the invariant extension property, i.e., every type over an algebraically closed set of parameters has a global invariant extension.

Proof. Weak elimination of imaginaries follows from Theorem 4.21 using Proposition 4.17. Finite sets are coded by Lemma 3.8. Elimination of imaginaries follows.

The invariant extension property follows again from the same theorem, using Proposition 4.22.

Remark 4.24. When a theory $T$ is NIP and eliminates imaginaries, as is the case for $\mathrm{SCVH}_{p, e}^{\mathcal{G}}$, the invariant extension property has various model theoretic consequences (cf. [16, Proposition 2.11]):

- Lascar strong type, Kim-Pillay strong type and strong type coincide. That is, over an algebraically closed set $A$, two points have the same type if and only if there exists a model containing $A$ over which they have the same type.

- Every algebraically closed set is an extension base and thus, by [2], forking coincides with dividing in $T$.

In fact, since non-forking types are Lascar invariant in NIP theories, the invariant extension property is equivalent to the conjunction of the above two conditions.

\section{Algebraic and Definable Closure}

In this section, we wish to describe the algebraic and definable closure in $\mathrm{SCVF}_{p, e}$ and $\mathrm{SCVH}_{p, e}$. Our main result is that they are no larger than what could be expected: they are the (relative) algebraic and definable closure in ACVF of the structure generated by the parameters. We will denote by $\langle A\rangle_{\lambda}$ (respectively $\langle A\rangle_{D}$ ) the $\mathcal{L}_{\mathcal{G}, p, e^{- \text {structure }}}^{\lambda}$ (respectively $\mathcal{L}_{\mathcal{G}, p, e^{-}}^{D}$-structure) generated by $A$.

Lemma 5.1. Let $M \models \operatorname{SCVF}_{p, e}$ and $A \leq \mathbf{V F}(M)$ (in $\mathcal{L}_{\text {div }, p, e}^{\lambda}$ ). Then

$$
\mathbf{V F}(\operatorname{acl}(A)) \subseteq \bar{A} .
$$

Proof. Let us first assume that $\operatorname{val}(A) \neq 0$. Since $A$ is closed under $\lambda$-functions, the extension $A \subseteq \mathbf{V F}(M)$ is separable and, as $\mathbf{V F}(M)$ is separably closed, $\bar{A}^{\text {sep }} \subseteq M$. As $A$ contains a $p$-basis of $\mathbf{V F}(M), \bar{A}^{\text {sep }}$ and $\mathbf{V F}(M)$ have the same imperfection degree and hence $\bar{A}^{\text {sep }} \models \mathrm{SCVF}_{p, e}$. By model completeness, $\mathbf{V F}(\operatorname{acl}(A)) \subseteq \bar{A}^{\text {sep }} \subseteq$ $\bar{A}$.

Now, if $\operatorname{val}(A)=0$, assume that $M$ is sufficiently saturated and let $c \in \mathbf{V F}_{p^{\infty}}(M)$ be transcendental over $\mathbf{V F}(\operatorname{acl}(A))$ and have positive valuation. By the previous 
paragraph, $\mathbf{V F}(\operatorname{acl}(A)) \subseteq \overline{\mathbf{V F}\left(\langle A c\rangle_{\lambda}\right)}=\overline{A(c)}$. Let $a \in \mathbf{V F}(\operatorname{acl}(A)) \subseteq \overline{A(c)}$. By construction, $c \notin \overline{A(a)}$ and hence $a \in \bar{A}$.

A similar argument allows us to reduce the study of $\operatorname{acl}_{\mathrm{SCVH}_{p, e}}$ on the field sort to the above result.

Lemma 5.2. Let $M \models \mathrm{SCVH}_{p, e}$ and $A \leq \mathbf{V F}(M)$ (in $\mathcal{L}_{\mathrm{div}, p, e}^{D}$ ). Then

$$
\mathbf{V F}(\operatorname{acl}(A)) \subseteq \bar{A} \text {. }
$$

Proof. Let $b$ be a canonical $p$-basis of $\mathbf{V F}(M)$ with $\operatorname{trdeg}(b / \mathbf{V F}(\operatorname{acl}(A)))=e$ (for example, $b$ is a very canonical $p$-basis over $\mathbf{V F}(\operatorname{acl}(A))$ ). As the $D_{i, n}(x)$ can be expressed as polynomials in $\lambda(x)$ and $b$, it follows that $\mathbf{V F}(\operatorname{acl}(A b)) \subseteq$ $\mathbf{V F}\left(\operatorname{acl}_{\operatorname{SCVF}_{p, e}}(A)\right) \subseteq \overline{\langle A\rangle_{\lambda}}$. The last inclusion is proved in Lemma 5.1. Moreover, by [30, Lemma 4.3], $\lambda(x)^{p}$ can be expressed as a polynomial in $D(x)$ and $b$ and hence $\langle A\rangle_{\lambda} \subseteq \overline{\langle A b\rangle_{D}}=\overline{A(b)}$. Let $a \in \mathbf{V F}(\operatorname{acl}(A)) \subseteq \overline{A(b)}$. By construction the tuple $b$ is algebraically independent from $a$ over $A$ and hence $a \in \bar{A}$.

Lemma 5.3. Let $T=\mathrm{SCVF}_{p, e}^{\mathcal{G}}$ or $T=\mathrm{SCVH}_{p, e}^{\mathcal{G}}, M \models T$ and $A \leq M$. Then

$$
\mathbf{V F}(\operatorname{acl}(A))=\mathbf{V F}(\operatorname{acl}(\mathbf{V F}(A)))=\overline{\mathbf{V F}(A)} \cap M .
$$

Proof. The second equality follows from Lemmas 5.1 and 5.2.

To prove the first equality it suffices to show that all definable functions from some $\mathbf{S}_{n}$ or $\mathbf{T}_{n}$ into $\mathbf{V F}$ have a finite image. It is enough to prove this for $T=$ $\mathrm{SCVF}_{p, e}^{\mathcal{G}}$. Consider $M \models \mathrm{SCVF}_{p, e}^{\mathcal{G}}$ of cardinality continuum that contains a dense countable subfield (for example ${\overline{\mathbb{F}_{p}\left(t_{i} \mid 0<i<e\right)\left(\left(t_{0}\right)\right)^{\mathrm{s}}}}^{\mathrm{sep}}$ ). In such a model, the sorts $\mathbf{S}_{n}$ and $\mathbf{T}_{n}$ are countable but any definable subset $\mathbf{X}$ of (some Cartesian power of) $\mathbf{V F}(M)$ is either finite or has cardinality continuum. To prove that last statement, taking a $\lambda$-resolution we may assume that $\mathbf{X}$ is quantifier-free $\mathcal{L}_{\text {div }}(M)$ definable. If it is not finite then, by Corollary 3.7, there is a a semialgebraic subset $\mathbf{X}^{\prime} \subseteq \mathbf{X}$ which is in $K$-definable bijection with $\mathcal{O}^{d}(K)$ for some $d>0$. It follows that $\mathbf{X}^{\prime}$ and thus $\mathbf{X}$ has cardinality continuum.

As functions with countable domain cannot have an image with cardinality continuum, it follows that any definable function from some $\mathbf{S}_{n}$ or $\mathbf{T}_{n}$ into $\mathbf{V F}$ has a finite image and hence $\mathbf{V F}(\operatorname{acl}(A)) \subseteq \operatorname{acl}(\mathbf{V F}(A))$.

Proposition 5.4. Let $T=\mathrm{SCVF}_{p, e}^{\mathcal{G}}$ or $T=\mathrm{SCVH}_{p, e}^{\mathcal{G}}, M \models T$ and $A \leq M$.

$$
\operatorname{acl}_{T}(A)=\operatorname{acl}_{\mathrm{ACVF}_{p, p}^{\mathcal{G}}}(A) \cap M
$$

Proof. Pick $a \in \operatorname{acl}_{T}(A)$. If $a \in \mathbf{V F}$, by Lemma 5.3, $a \in \overline{\mathbf{V F}(A)} \subseteq \operatorname{acl}_{\mathrm{ACVF}_{p, p}^{\mathcal{G}}}(A)$.

Let us now assume that $a \in \mathbf{S}_{n}$ (the same proof also works if $a \in \mathbf{T}_{n}$ ). By quantifier elimination in the geometric language, there is a quantifier free $\mathcal{L}_{\mathcal{G}}(A)$ formula $\varphi(x)$ such that $\varphi(M)$ is finite and $M \models \varphi(a)$. As $\mathbf{S}_{n}(\bar{M})=\mathbf{S}_{n}(M)$ and $\varphi(x)$ is quantifier free, we have $a \in \varphi(M)=\varphi(\bar{M})$, with $\varphi(\bar{M})$ finite. It follows that $a \in \operatorname{acl}_{\mathrm{ACVF}_{p, p}^{\mathcal{G}}}(A)$.

Proposition 5.5. Let $T=\mathrm{SCVF}_{p, e}^{\mathcal{G}}$ or $T=\mathrm{SCVH}_{p, e}^{\mathcal{G}}, M \models T$ and $A \leq M$.

$$
\operatorname{dcl}_{T}(A)=\operatorname{dcl}_{\mathrm{ACVF}_{p, p}^{\mathcal{G}}}(A) \cap M
$$


Proof. Pick $a \in \operatorname{dcl}_{T}(A)$. If $a \in \mathbf{S}_{n}$ or $\mathbf{T}_{n}$, then, as above, there is a quantifier free $\mathcal{L}_{\mathcal{G}}(A)$-formula $\varphi(x)$ such that $\varphi(M)=\{a\}=\varphi(\bar{M})$ and thus $a \in \operatorname{dcl}_{\mathrm{ACVF}_{p, p}^{\mathcal{G}}}(A)$.

If $a \in \mathbf{V F}$, by Lemma 5.3, $a \in \overline{\mathbf{V F}(A)} \cap M=: F$. Let $\sigma \in \operatorname{Aut}_{\mathrm{ACVF}_{p, p}^{\mathcal{G}}}(\bar{M} / A)$. The Hasse derivations (and hence the $\lambda$-functions) extend uniquely from $\mathbf{V} \mathbf{F}(A)$ to $F$. It follows that $\left.\sigma\right|_{A \cup F}$ respects all the new structure on $\mathbf{V F}$ in $T$ and therefore $\sigma(a)$ is a $T$-conjugate of $a$ over $A$. In particular $\sigma(a)=a$ and $a \in \operatorname{dcl}_{\mathrm{ACVF}_{p, p}^{\mathcal{G}}}(A)$.

\section{Metastability}

In this section, we will show that $\mathrm{SCVF}_{p, e}$ is metastable (as defined by Haskell, Hrushovski and Macpherson in [9]). But first, let us give some definitions.

An $\mathcal{L}(A)$-definable set $\mathbf{X}$ is said to be stably embedded if every $\mathcal{L}(M)$-definable set $\mathbf{Y} \subseteq \mathbf{X}^{n}$ is $\mathcal{L}(A \cup \mathbf{X}(M))$-definable. The set $\mathbf{X}$ is said to be stable (if it is stably embedded and) if the structure on $\mathbf{X}$ induced by $\mathcal{L}(A)$ is stable. For a thorough discussion of (stably embedded) stable sets, we refer the reader to the appendix of [1]. We denote by $\mathrm{St}_{A}$ the structure whose sorts are the stable (stably embedded) sets which are $\mathcal{L}(A)$-definable, equipped with their $\mathcal{L}(A)$-induced structure. We will denote by $\downarrow_{A}$ forking independence in $\mathrm{St}_{A}$.

Lemma 6.1. Let $T=\mathrm{SCVH}_{p, e}$ or $T=\mathrm{SCVF}_{p, e}$, and let $\mathbf{X}$ be an infinite definable subset of $\mathbf{V F}^{n}$ for some $n \in \mathbb{N}$. Then there is a definable function $f: \mathbf{X} \rightarrow \boldsymbol{\Gamma}$ with infinite image. In particular, $\mathbf{X}$ is unstable.

Proof. We may work over parameters, and it is thus enough to prove the result for $T=\mathrm{SCVF}_{p, e}$. Assume $\mathbf{X}$ is defined over $K \models \mathrm{SCVF}_{p, e}$. Using $\lambda$-resolutions, we may assume that $\mathbf{X}$ is a semialgebraic subset of $K^{n}$, i.e. defined by a quantifier-free $\mathcal{L}_{\text {div }}(K)$-formula. By Corollary 3.7, there is a a semialgebraic subset $\mathbf{X}^{\prime} \subseteq \mathbf{X}$ which is in $K$-definable bijection with $\mathcal{O}_{K}^{d}$, where $d>0$ is the dimension of the Zariski closure of $\mathbf{X}$. The result follows, by considering the function $f: \mathcal{O}_{K}^{d} \rightarrow \boldsymbol{\Gamma}$ sending $x$ to $\operatorname{val}\left(x_{1}\right)$.

Proposition 6.2. Let $T=\mathrm{SCVH}_{p, e}^{\mathcal{G}}$ or $T=\mathrm{SCVF}_{p, e}^{\mathcal{G}}$, and let $A \leq M \models T$. Suppose that $\mathbf{X}$ is an A-definable set. Then the following are equivalent:

(1) $\mathbf{X}$ is stable stably embedded.

(2) $\mathbf{X}$, expanded by relations for A-definable subsets of $\mathbf{X}^{n}$ for all $n$, has finite Morley rank.

(3) $\mathbf{X} \perp \boldsymbol{\Gamma}$, i.e., any definable subset of $\mathbf{X}^{n} \times \boldsymbol{\Gamma}^{m}$ is a finite union of rectangles.

(4) There is no definable function $f: \mathbf{X} \rightarrow \boldsymbol{\Gamma}$ with infinite image.

(5) $\mathbf{X}$ is $\mathbf{k - i n t e r n a l . ~}$

(6) $\mathbf{X}$ is k-analyzable.

(7) Possibly after a permutation of coordinates, $\mathbf{X}$ is contained in a finite union of sets of the form $s_{1} / \mathfrak{m} s_{1} \times \cdots \times s_{m} / \mathfrak{m} s_{m} \times F$, where the $s_{i}$ are $\operatorname{acl}_{T}(A)$ definable lattices and $F$ is an A-definable finite set of tuples from $\mathcal{G}(M)$.

Proof. The same characterization of stable stably embedded definable sets holds in ACVF by [8, Lemma 2.6.2]. Note that (3) and (4) are equivalent, since $\boldsymbol{\Gamma}$ is stably embedded in $T$ and eliminates imaginaries. The characterization thus holds in $T$ for any $A$-definable set $\mathbf{X}$ which lives in $\mathcal{G}^{\text {im }}$, by Corollary 2.18 .

Now let $\mathbf{X}$ be a definable subset of $\mathbf{V F}^{n} \times G$, where $G$ is a finite product of sorts from $\mathcal{G}^{\text {im }}$. If the projection of $\mathbf{X}$ to $\mathbf{V} \mathbf{F}^{n}$ is infinite, the negation of (4) holds 
by Lemma 6.1, and the negation of all other statements follows easily from this. We may thus assume that the projection $F$ of $\mathbf{X}$ to $\mathbf{V F}^{n}$ is finite, and so we are reduced to definable subsets of $\mathcal{G}^{\text {im }}$.

Definition 6.3 (Stable domination). Let $M$ be some $\mathcal{L}$-structure, $C \subseteq M$, $f$ a pro-definable map to $\mathrm{St}_{C}$ (defined with parameters in $C$ ) and $a \in M$. We say that $\operatorname{tp}(a / C)$ is stably dominated via $f$ if for every $a \models p$ and $B \subseteq M$ such that $\operatorname{St}_{C}(\operatorname{dcl}(C B)) \downarrow_{C} f(a)$,

$$
\operatorname{tp}(B / C f(a)) \vdash \operatorname{tp}(B / C a) .
$$

We say that $p$ is stably dominated if it is stably dominated via some map $f$.

Note that if $p$ is stably dominated, it is stably dominated via any map enumerating $\operatorname{St}_{C}(\operatorname{dcl}(C a))$ for any $a \models p$.

Remark 6.4. In the definition of stable domination, it suffices to show that for any $B$ there exists $B^{\prime}$ such that $B \subseteq \operatorname{dcl}\left(B^{\prime}\right)$ and if $\operatorname{St}_{C}\left(\operatorname{dcl}\left(C B^{\prime}\right)\right) \downarrow_{C} f(a)$, then $\operatorname{tp}\left(B^{\prime} / C f(a)\right) \vdash \operatorname{tp}\left(B^{\prime} / C a\right)$.

Indeed, as $\mathrm{St}_{C}$ is stably dominated, if $\operatorname{St}_{C}(\operatorname{dcl}(C B)) \downarrow_{C} f(a)$, we may also assume (taking a conjugate of $B^{\prime}$ over $B$ ) that $\operatorname{St}_{C}\left(\operatorname{dcl}\left(C B^{\prime}\right)\right) \downarrow_{C} f(a)$ and if $\operatorname{tp}\left(B^{\prime} / C f(a)\right) \vdash \operatorname{tp}\left(B^{\prime} / C a\right)$, then $\operatorname{tp}(B / C f(a)) \vdash \operatorname{tp}(B / C a)$.

Definition 6.5 (Metastability). Let $T$ be a theory and $\boldsymbol{\Gamma}$ an $\emptyset$-definable stably embedded set. We say that $T$ is metastable over $\boldsymbol{\Gamma}$ if:

(1) The theory $T$ has the invariant extension property (as in Corollary 4.23).

(2) For $M \models T$ sufficiently saturated and for every small subset $A \subseteq M$, there exists a small subset $C \subseteq M$ containing $A$ such that for all tuples $a \in M$, $\operatorname{tp}(a / C \boldsymbol{\Gamma}(\operatorname{dcl}(C a)))$ is stably dominated.

Such a $C$ is called a metastability basis.

Let $T$ be a theory and $U \models T$ a monster model of $T$. Let $p(x), q(y) \in S(U)$ be definable types. Then one may define the tensor product $(p \otimes q)(x, y) \in S(U)$ as follows. Let $C \subseteq U$ be small such that $p$ and $q$ are $C$-definable. Then $p \otimes q$ is the unique $C$-definable type in $S(U)$ satisfying

$$
(a, b) \models(p \otimes q) \mid B \text { if and only if } a \models p \mid B b \text { and } b \models q \mid B
$$

for all small $B \subseteq U$ containing $C$ and all $(a, b) \in U$. We refer to [27] for the basic properties of definable types, $\otimes$ and generically stable types which we will define now.

Definition 6.6. Let $T$ be NIP and $U \models T$ a monster model. An invariant type $p(x) \in S(U)$ is called generically stable if $p(x) \otimes p(y)=p(y) \otimes p(x)$.

Let us also define orthogonality.

Definition 6.7. Let $Q$ be an $\emptyset$-definable stably embedded set. A type $p \in S(C)$ is said to be almost orthogonal to $Q$ if $\operatorname{dcl}(C a) \cap Q^{\text {eq }}=\operatorname{dcl}(C) \cap Q^{\text {eq }}$ for any $a \models p$. An invariant type $p \in S(U)$ is orthogonal to $Q$, denoted by $p \perp Q$, if $p \mid B$ is almost orthogonal to $Q$ for every set $B \subseteq U$ over which $p$ is invariant.

As a consequence of metastability (and NIP), we get an alternative characterization of stably dominated types in case $\boldsymbol{\Gamma}$ is totally ordered. 
Proposition 6.8. Let $T$ be an NIP theory which is metastable over the stably embedded $\emptyset$-definable set $\boldsymbol{\Gamma}$. Assume that $\boldsymbol{\Gamma}$ admits a definable total ordering and eliminates imaginaries. A global invariant type $p \in S(U)$ is stably dominated if and only if $p$ is generically stable if and only if $p \perp \Gamma$.

Proof. In [14, Proposition 2.9.1.a], Hrushovski and Loeser show that the above equivalences hold in ACVF. The proof given there generalizes easily to this more abstract setting.

In [9], Haskell, Hrushovski and Macpherson showed that maximally complete fields are metastability bases in ACVF. In $\mathrm{SCVF}_{p, e}$ and $\mathrm{SCVH}_{p, e}$, we will prove that the situation is quite similar: separably maximally complete fields are metastability bases.

But first, let us characterize stably dominated types in $\mathrm{SCVH}_{p, e}$. As $\mathrm{SCVF}_{p, e}$ is an enrichment of $\mathrm{SCVH}_{p, e}$ by constants, the same results will follow for $\mathrm{SCVF}_{p, e}$. We extend $D_{\omega}$ to all of $\mathcal{G}$ by setting $D_{\omega}(a)=a$ for non field points.

Proposition 6.9. Let $M \models \mathrm{SCVH}_{p, e}^{\mathcal{G}}, C \subseteq M$ be closed under $D, a \in M$ a tuple and $f$ a pro-definable function. The following are equivalent:

(i) The type $\operatorname{tp}_{M}(a / C)$ is stably dominated via $f$ (in $M$ ).

(ii) There exists, in $\bar{M}$, a pro-definable function $g$ such that $f=g \circ D_{\omega}$ and the type $\operatorname{tp}_{\bar{M}}\left(D_{\omega}(a) / C\right)$ is stably dominated via $g$ (in $\left.\bar{M}\right)$.

Proof. First, note that the existence of $g$ follows immediately from Proposition 5.5.

By Remark 6.4, to prove stable domination of $\operatorname{tp}_{M}(a / C)$ and $\operatorname{tp}_{\bar{M}}\left(D_{\omega}(a) / C\right)$, it suffices to consider $B=\mathbf{V F}\left(\operatorname{dcl}_{M}(C B)\right) \subseteq \mathbf{V F}(M)$.

Moreover, for such a $B$, we have that $\operatorname{St}_{C}^{M}(B) \downarrow_{C}^{M} g\left(D_{\omega}(a)\right)$ if and only if $\mathrm{St}_{C}^{\bar{M}}\left(\operatorname{dcl}_{\bar{M}}(B)\right) \downarrow_{C}^{\bar{M}} g\left(D_{\omega}(a)\right)$. Indeed, by Proposition 6.2, $\mathrm{St}_{C}^{M}$ and $\mathrm{St}_{C}^{\bar{M}}$ are essentially the same structure up to the fact that that $\mathrm{St}_{C}^{\bar{M}}$ has some more finite sorts that are irrelevant to forking. Also, by quantifier elimination, $\operatorname{tp}_{M}(B / C a)$ is equivalent to $\operatorname{tp}_{\bar{M}}\left(B / C D_{\omega}(a)\right)$ and $\operatorname{tp}_{M}(B / C f(a))$ is equivalent to $\operatorname{tp}_{\bar{M}}\left(B / C g\left(D_{\omega}(a)\right)\right)$ (note that we are implicitly using the fact that $C g\left(D_{\omega}(a)\right)$ is closed under $D$ as the image of $g$ is in $\mathrm{St}_{C}$ ).

The equivalence of (i) and (ii) is an immediate consequence.

Proposition 6.10. Let $M \models \mathrm{SCVH}_{p, e}, C \leq \mathbf{V F}(M)$ be separably maximally complete and $a \in M$ be a tuple, then $\operatorname{tp}(a / C \boldsymbol{\Gamma}(\operatorname{dcl}(C a)))$ is stably dominated.

Proof. Let

$$
\begin{gathered}
E=C \boldsymbol{\Gamma}\left(\operatorname{dcl}_{\bar{M}}\left(C D_{\omega}(a)\right)\right)=C \boldsymbol{\Gamma}\left(\operatorname{dcl}_{M}(C a)\right) \\
\bar{E}=E \cup \bar{C}^{\text {perf }} \\
g\left(D_{\omega}(a)\right)=\operatorname{St}_{E}^{M}\left(\operatorname{dcl}_{M}\left(E D_{\omega}(a)\right)\right) \\
\bar{g}\left(D_{\omega}(a)\right)=\operatorname{St}_{\frac{M}{E}}\left(\operatorname{dcl}_{\bar{M}}\left(\bar{E} D_{\omega}(a)\right)\right)=g\left(D_{\omega}(a)\right) \cup \bar{C}^{\text {perf }}
\end{gathered}
$$

By Lemma $4.15, \bar{C}^{\text {perf }}$ is maximally complete. Thus, $\operatorname{tp}_{\bar{M}}\left(D_{\omega}(a) / \bar{E}\right)$ is stably dominated via $\bar{g}$ by $\left[9\right.$, Theorem 12.18.(ii)], and hence $\operatorname{tp}_{\bar{M}}\left(D_{\omega}(a) / E\right)$ is stably dominated via $g$. By Proposition $6.9, \operatorname{tp}_{M}(a / E)=\operatorname{tp}(a / C \boldsymbol{\Gamma}(\operatorname{dcl}(C a)))$ is also stably dominated.

Corollary 6.11. The theories $\mathrm{SCVH}_{p, e}$ and $\mathrm{SCVF}_{p, e}$ are metastable over $\boldsymbol{\Gamma}$. 


\section{The Stable Completion of a Definable Set in SCVF}

The goal of this section is to generalize a result of Hrushovski and Loeser [14] on the strict pro-definability of the space of stably dominated types. We show that their proof holds in a context general enough to also encompass separably closed valued fields of finite imperfection degree and Scanlon's theory of contractive valued differential fields (see [25]).

For a proof of the following result, see, e.g., [27, Remark 2.32].

Fact 7.1. Let $T$ be NIP and $U \models T$. Then generically stable types are uniformly definable in $T$ : for any formula $\varphi(x ; y)$ there is a formula $\theta(y ; z)$ such that for every generically stable type $p(x) \in S(U)$ there is $b \in U$ such that $d_{p} x \varphi(x ; y)=\theta(y, b)$.

Hrushovski and Loeser [14] use this fact, together with Proposition 6.8, to encode the set $\widehat{\mathbf{X}}$ of global stably dominated types concentrating on some definable set $\mathbf{X}$ in ACVF as a pro-definable set.

Notation. Given an $\emptyset$-definable stably embedded set $\mathbf{Q}$, a $C$-definable set $\mathbf{X}$ and a set $A$ containing $C, S_{\text {def, } \mathbf{X}}(A)$ denotes the set of global $A$-definable types $p(x)$ such that $p(x) \vdash x \in \mathbf{X}$, and $S_{\text {def, } \mathbf{X}}^{\mathbf{Q}}(A):=\left\{p \in S_{\mathrm{def}, \mathbf{X}}(A) \mid p \perp \mathbf{Q}\right\}$. Finally, $\widehat{\mathbf{X}}(A):=\left\{p \in S_{\text {def }, \mathbf{X}}(A) \mid p\right.$ is stably dominated $\}$.

Fact 7.2 ([14, Lemma 2.5.1]). Assume $T$ eliminates imaginaries. Let $\mathbf{Q}$ be an $\emptyset$-definable stably embedded set. Assume that the definable types orthogonal to $\mathbf{Q}$ are uniformly definable. Then for any $C$-definable set $\mathbf{X}, S_{\mathrm{def}, \mathbf{X}}^{\mathbf{Q}}$ is $C$-pro-definable: there is a $C$-pro-definable set $\mathbf{Z}$ such that for any $A \supseteq C$ there is a canonical bijection $\mathbf{Z}(A) \simeq S_{\mathrm{def}, \mathbf{X}}^{\mathbf{Q}}(A)$.

Moreover, if $f: \mathbf{X} \rightarrow \mathbf{Y}$ is a definable function, then, identifying $S_{\mathrm{def}, \mathbf{X}}^{\mathbf{Q}}$ and $S_{\mathrm{def}, \mathbf{Y}}^{\mathbf{Q}}$ with the corresponding pro-definable sets, the map $f_{\mathrm{def}, \mathbf{X}}: S_{\mathrm{def}, \mathbf{X}}^{\mathbf{Q}} \rightarrow S_{\mathrm{def}, \mathbf{Y}}^{\mathbf{Q}}$, $p \mapsto f_{\star}(p)$ is pro-definable.

We briefly sketch the argument. For notational simplicity, we will assume $C=\emptyset$. Let $f: \mathbf{X} \rightarrow \mathbf{Q}^{\text {eq }}$ be definable (with parameters) and let $p \in S_{\text {def, } \mathbf{X}}^{\mathbf{Q}}(U)$. As $p \perp \mathbf{Q}$, $f_{\star}(p)$ is a realized type, i.e., there is $\gamma \in \mathbf{Q}^{\mathrm{eq}}$ such that $\mathrm{d}_{p} x(f(x)=\gamma)$. We will denote this by $p_{\star}(f)=\gamma$.

Now let $f: \mathbf{X} \times \mathbf{W} \rightarrow \mathbf{Q}^{\text {eq }}$ be $\emptyset$-definable, $f_{w}:=f(-, w)$. It follows from the assumptions that there is a set $\mathbf{S}$ and a function $g: \mathbf{S} \times \mathbf{W} \rightarrow \mathbf{Q}^{\mathrm{eq}}$, both $\emptyset$-definable, such that for every $p \in S_{\operatorname{def}, \mathbf{X}}^{\mathbf{Q}}(U)$, the function

$$
p_{\star}(f): \mathbf{W} \rightarrow \mathbf{Q}^{\mathrm{eq}}, w \mapsto p_{\star}\left(f_{w}\right)
$$

is equal to $g_{s}=g(s,-)$ for a unique $s \in \mathbf{S}$.

Now choose an enumeration $f_{i}: \mathbf{X} \times \mathbf{W}_{i} \rightarrow \mathbf{Q}^{\mathrm{eq}}(i \in I)$ of the functions as above (with corresponding $g_{i}: S_{i} \times \mathbf{W}_{i} \rightarrow \mathbf{Q}^{\mathrm{eq}}$ ). Then

$$
p \mapsto c(p):=\left\{\left(s_{i}\right)_{i \in I} \mid p_{\star}\left(f_{i}\right)=g_{i, s_{i}} \text { for all } i\right\}
$$

defines an injection of $S_{\mathrm{def}, \mathbf{X}}^{\mathbf{Q}}$ into $\prod_{i \in I} \mathbf{S}_{i}$, and one may show that the image $\mathbf{Y}_{i}$ of $c\left(S_{\mathrm{def}, \mathbf{X}}^{\mathbf{Q}}\right)$ under the projection map to $\mathbf{S}_{i}$ is $\infty$-definable. Since the set of $\mathbf{S}_{i}$ 's is closed under taking finite products (this may be seen using products of the corresponding $f_{i}$ 's $)$, pro-definability of $c\left(S_{\mathrm{def}, \mathbf{X}}^{\mathbf{Q}}\right)$ follows. 
Corollary 7.3. Let $T$ be a theory which eliminates imaginaries, and let $\mathbf{X}$ be a $C$-definable set.

(1) Assume $T$ is stable. Then $S_{\text {def, } \mathbf{X}}$ is canonically a $C$-pro-definable set.

(2) Assume $T$ is NIP and metastable over the stably embedded $\emptyset$-definable set $\boldsymbol{\Gamma}$. Assume that $\boldsymbol{\Gamma}$ admits a definable total ordering and eliminates imaginaries. Then $\widehat{\mathbf{X}}$ is canonically C-pro-definable. Moreover, if $f: \mathbf{X} \rightarrow \mathbf{Y}$ is a definable function, then the map $\widehat{f}: \widehat{\mathbf{X}} \rightarrow \widehat{\mathbf{Y}}, p \mapsto f_{\star}(p)$ is pro-definable, once $\widehat{\mathbf{X}}$ and $\widehat{\mathbf{Y}}$ are identified with the corresponding pro-definable sets.

Proof. Both parts follow from Fact 7.2. For (1), note that if $\mathbf{Q}$ is a 2-element set, then $S_{\text {def, } \mathbf{X}}^{\mathbf{Q}}=S_{\text {def, } \mathbf{X}}$. Since in a stable theory all definable types are generically stable, uniform definability of types follows from Fact 7.1.

In (2), one has $\widehat{\mathbf{X}}(A)=S_{\text {def, } \mathbf{X}}^{\Gamma}(A)$ for all $A \supseteq C$, by Proposition 6.8. As in the proof of (1), uniform definability holds by Fact 7.1.

Recall that a theory $T$ has the finite cover property if there is a formula $\varphi(x, y)$ (where $x$ and $y$ may be tuples of variables) such that for every $n \in \mathbb{N}$ there are $a_{1}, \ldots, a_{n} \in U \models T$ such that $\models \neg \exists x \bigwedge_{i \leq n} \varphi\left(x, a_{i}\right)$ and $\models \exists x \bigwedge_{i \leq n, i \neq k} \varphi\left(x, a_{i}\right)$ for every $k \leq n$. The theory $T$ is $n f c p$ if it does not have the finite cover property. By a result of Shelah [26, II Theorems 4.2, 4.4], $T$ is nfcp if and only if $T$ is stable and $T^{\mathrm{eq}}$ eliminates $\exists^{\infty} x$.

The following characterization is due to Poizat.

Fact 7.4 ([21, Théorème5]). Let $T$ be stable. Then $T$ is nfcp if and only if for every pair of formulas $\varphi(x ; y)$ and $\theta(y ; z)$ the set

$$
\mathbf{D}_{\varphi, \theta}=\{c \in U \mid \theta(y, c) \text { is the } \varphi \text {-definition of a (complete) global type }\}
$$

is definable.

Corollary 7.5. Let $T$ be stable. Then $T$ is $n f c p$ if and only if for every definable set $\mathbf{X}$, the set $S_{\mathrm{def}, \mathbf{X}}$ is strict pro-definable.

Proof. Let $\mathbf{Z}=\lim \mathbf{Z}_{i}$ be the pro-definable set given by the proof of Fact 7.2, with $\mathbf{Z}(A) \simeq S_{\text {def, } \mathbf{X}}(A)$ canonically. Then $\mathbf{Z}_{i}$ corresponds to canonical parameters of instances of a formula $\theta=\theta_{\varphi}(y ; z)$, and $\pi_{i}(\mathbf{Z}) \subseteq \mathbf{Z}_{i}$ is precisely the set of those parameters corresponding to $\varphi$-definitions of complete global types, i.e., $\pi_{i}(\mathbf{Z})=$ $\mathbf{D}_{\varphi, \theta}$. We conclude by Fact 7.4.

Hrushovski and Loeser showed that for every definable set $\mathbf{X}$ in ACVF, the set $\widehat{\mathbf{X}}$ of stably dominated types concentrated on $\mathbf{X}$ is strict pro-definable $[14$, Theorem 3.1.1]. Analyzing their proof, we obtain the following abstract version of this important technical result, which covers the theories we are interested in.

Theorem 7.6. Let $T$ be a complete theory which eliminates imaginaries, is NIP and metastable over some $\emptyset$-definable stably embedded set $\boldsymbol{\Gamma}$. Let $\mathbf{k}_{i \in I}$ be $\emptyset$-definable stable stably embedded sets. Assume the following properties hold:

(1) $\boldsymbol{\Gamma}$ eliminates imaginaries and admits a definable total ordering;

(2) every set of parameters $A$ is included in a metastability basis $C$ such that for every parameter set $B=\operatorname{dcl}(C B), \mathrm{St}_{C}(B)$ is interdefinable with $\bigcup_{i} \mathbf{k}_{i}(B)$;

(3) The (multisorted) structure $\bigcup_{i} \mathbf{k}_{i}$ (with the induced structure) is nfcp.

Then, for every A-definable set $\mathbf{X}$, the set $\widehat{\mathbf{X}}$ is strict A-pro-definable. 
Proof. Let $\mathbf{X}$ be $A$-definable. Pro-definability of $\widehat{\mathbf{X}}$ is the content of Corollary 7.3(2). We recall the construction of the pro-definable set $\mathbf{D}$ satisfying $\mathbf{D}(B)=$ $\widehat{\mathbf{X}}(B)$ canonically, for every parameter set $B \supseteq A$. Let $f_{i}: \mathbf{X} \times \mathbf{W}_{i} \rightarrow \Gamma^{n_{i}}(i \in I)$ be an enumeration of all $A$-definable families of functions from $\mathbf{X}$ to $\Gamma^{\mathrm{eq}}$. As $\boldsymbol{\Gamma}$ eliminates imaginaries, it is enough to consider functions with values in $\boldsymbol{\Gamma}^{n}$ for some $n$. Let $g_{i}: \mathbf{S}_{i} \times \mathbf{W}_{i} \rightarrow \Gamma^{n_{i}}$ be $A$-definable such that for any $p \in \widehat{\mathbf{X}}(U)$ the function $p_{\star}\left(f_{i}\right): \mathbf{W}_{i} \rightarrow \Gamma^{n_{i}}$ is equal to $g_{i, s_{i}}=g_{i}\left(s_{i},-\right)$ for some unique $s_{i} \in \mathbf{S}_{i}$. Then $\mathbf{D}$ is the image of the injective map $c: \widehat{\mathbf{X}} \hookrightarrow \prod_{i \in I} \mathbf{S}_{i}, p \mapsto\left(s_{i}\right)_{i \in I}$.

In order to show that $\mathbf{D}$ is strict pro-definable, it is enough to show that the projection $\mathbf{Y}_{i}:=\pi_{i}(\mathbf{D}) \subseteq \mathbf{S}_{i}$ is definable for all $i \in I$. (Note that the $\mathbf{S}_{i}$ 's are closed under taking finite products.) We already know that $\mathbf{Y}_{i}$ is $\infty$-definable, so there only remains to show that it is a union of definable sets, i.e., ind-definable. Now fix $i \in I$. We will omit indices and write $f: \mathbf{X} \times \mathbf{W} \rightarrow \Gamma^{n}$ and $g: \mathbf{S} \times \mathbf{W} \rightarrow \boldsymbol{\Gamma}^{n}$ in what follows.

Let $\mathbf{Z}$ be the set of functions $g_{0}: \mathbf{W} \rightarrow \boldsymbol{\Gamma}^{n}$ such that there exist:

- a finite product $\mathbf{L}=\prod_{j} \mathbf{k}_{j}^{n_{j}}$;

- a function $h: \mathbf{X} \rightarrow \mathbf{L}$, definable with parameters $c$, and

- for $\varphi(y ; c, v, w)=(\exists x \in \mathbf{X} h(x)=y) \wedge(\forall x \in \mathbf{X} h(x)=y \rightarrow f(x, w)=v)$, a definable $\varphi(y ; c, v, w)$-type $q_{0}$ concentrating on $\mathbf{L}$

satisfying $g_{0}(w)=\gamma$ if and only if $d_{q_{0}} y \varphi(y ; c, \gamma, w)$.

By Fact 7.4 , for every formula $\theta(v, w ; z)$, the fact that, for a given $t, \theta(v, w, t)$ is the $\varphi(y ; c, v, w)$-definition of a consistent type is a definable condition in $t$. It follows that $\mathbf{Z}$ is ind-definable. We will now show that $\mathbf{Z}=\mathbf{Y}$.

First, pick $p \in \widehat{\mathbf{X}}$. Let $C$ be a set as in Hypothesis 2 such that $C \supseteq A$ and $p$ is $C$-definable. By stable domination, Hypothesis 2 (and compactness), there exists $h$ and $\mathbf{L}$ as above such that for all $\left.a \models p\right|_{C}$ and all $b$ and $\gamma$, if $h(a) \downarrow_{C} \operatorname{St}_{C}(\operatorname{dcl}(C b \gamma))$, $\operatorname{tp}(b, \gamma, h(a)) \vdash f(a, b)=\gamma$. Actually, making $h$ bigger we may assume that the above holds for all $a \in \mathbf{X}$. Let $q=h_{\star} p$, then $\left.q\right|_{C b \gamma}(y) \vdash \forall x \in \mathbf{X} h(x)=y \rightarrow$ $f(x, b)=\gamma$ and the tuple $\left(\mathbf{L}, h,\left.q\right|_{\theta}\right)$ proves that $p_{\star} f \in \mathbf{Z}$ and hence $\mathbf{Y} \subseteq \mathbf{Z}$.

Now, let $g_{0} \in \mathbf{Z}$ and $\mathbf{L}, h$ and $q_{0}$ as in the definition of $\mathbf{Z}$. Let $C$ be as in Hypothesis 2, such that $g_{0}, h$ and $q_{0}$ are defined over $C$. Let $\left.b \models q_{0}\right|_{C}$ and $a \in \mathbf{X}$ such that $h(a)=b$. Let $C^{\prime}=\operatorname{acl}(C \boldsymbol{\Gamma}(\operatorname{dcl}(C a)))$. Since $C$ is a metastability basis, $\operatorname{tp}\left(a / C^{\prime}\right)$ is stably dominated (and thus in particular definable over $C^{\prime}$ ), so $h_{\star} p$ is definable over $C^{\prime}$. By orthogonality of $\boldsymbol{\Gamma}$ and the stable part, $h_{\star} p$ is definable over $\mathrm{St}_{C}\left(C^{\prime}\right)=\mathrm{St}_{C}(C)$, which is interdefinable with $C$. As $h_{\star} p$ extends $\left.q_{0}\right|_{C}$, we have that $h_{\star} p=q_{0}$. Let $b \in \mathbf{W}, \gamma=g_{0}(b)$ and $\left.a \models p\right|_{C^{\prime} b \gamma}$, then $\left.h(a) \models q_{0}\right|_{C b \gamma}$ and, by definition of $\mathbf{Z}, f(a, b)=\gamma$. It follows that $p_{\star} f(b)=g_{0}(b)$, hence $g_{0}=p_{\star} f \in \mathbf{Y}$ and so $\mathbf{Z} \subseteq \mathbf{Y}$.

Corollary 7.7. Let $T=\mathrm{SCVH}_{p, e}$ (or any completion of $\mathrm{SCVF}_{p, e}$ ). Then for every $A$-definable set $\mathbf{X}, \widehat{\mathbf{X}}$ may be canonically identified with a strict $A$-pro-definable set.

Proof. This follows from Theorem 7.6. Indeed, $\mathrm{SCVH}_{p, e}$ is NIP (Corollary 2.14), and it is metastable over $\boldsymbol{\Gamma}$ by Corollary 6.11. Since $\boldsymbol{\Gamma}$ is a stably embedded pure divisible ordered Abelian group, it eliminates imaginaries. Any parameter set $A$ is contained in a separably maximal model $K$, and such a $K$ is a metastability basis (see Proposition 6.10). 
Over $K$, indeed over any model of $\mathrm{SCVH}_{p, e}, \mathrm{St}_{K}(B)=\operatorname{dcl}(\mathbf{k}(B))$ for every parameter set $B=\operatorname{dcl}(B K)$. This follows from Proposition 6.2(7) and the fact that every lattice $s \in \mathbf{S}_{n}(K)$ has a $K$-definable basis. Since the residue field $\mathbf{k}$ is a pure model of $\mathrm{ACF}_{p}$, it is nfcp. (Note that purity and stable embeddedness of $\boldsymbol{\Gamma}$ and $\mathbf{k}$ follow from the corresponding results in $\mathrm{ACVF}_{p, p}$, by Corollary 2.18.) Thus, all hypotheses of Theorem 7.6 are satisfied.

We now discuss a similar context in which Theorem 7.6 applies. Let $\operatorname{VDF}_{\mathcal{E C}}$ be the theory of existentially closed valued differential fields $(K, v, \partial)$ of residue characteristic 0 satisfying $v(\partial(x)) \geq v(x)$ for all $x$. This theory had first been studied by Scanlon [25]. The theory $\operatorname{VDF}_{\mathcal{E C}}$ is NIP, and the residue field is stably embedded and a pure model of $\mathrm{DCF}_{0}$, with the derivation induced by $\partial$.

The third author has shown in [23] that $\mathrm{VDF}_{\mathcal{E C}}$ eliminates imaginaries in the geometric sorts and that it is metastable over the value group $\boldsymbol{\Gamma}$, a stably embedded pure divisible ordered Abelian group. As shown there, every set of parameters is included in a metastability basis which is a model, and over any model any stably embedded stable set is interdefinable with the residue field, since it is the case in the underlying algebraically closed valued field. As $\mathrm{DCF}_{0}$ is stable and eliminates imaginaries, it is enough to show that it eliminates $\exists^{\infty} x$. But this follows from the fact that the algebraic closure of a set $A$ is the field theoretic algebraic closure of the differential field generated by $A$, by quantifier elimination.

We have thus proved the following result.

Corollary 7.8. Let $\mathbf{X}$ be any A-definable set in a model of $\operatorname{VDF}_{\mathcal{E C}}$. Then $\widehat{\mathbf{X}}$ may be canonically identified with a strict A-pro-definable set.

\section{REFERENCES}

[1] Zoé Chatzidakis and Ehud Hrushovski. Model theory of difference fields. Trans. Amer. Math. Soc., 351(8):2997-3071, 1999.

[2] Artem Chernikov and Itay Kaplan. Forking and dividing in $\mathrm{NTP}_{2}$ theories. J. Symbolic Logic, 77(1):1-20, 2012.

[3] Françoise Delon. Idéaux et types sur les corps séparablement clos. Mém. Soc. Math. France (N.S.), 33:76, 1988.

[4] Françoise Delon. Separably closed fields. In Model theory and algebraic geometry, volume 1696 of Lecture Notes in Math., pages 143-176. Springer, Berlin, 1998.

[5] Françoise Delon. Quantifier elimination in pairs of algebraically closed fields. Confluentes Math., 4(2), 2012.

[6] Antoine Ducros. Les espaces de Berkovich sont modérés (d'après Ehud Hrushovski et François Loeser). Astérisque, 352:Exp. No. 1056, x, 459-507, 2013. Séminaire Bourbaki. Vol. 2011/2012. Exposés 1043-1058.

[7] Ju. L. Ershov. Fields with a solvable theory. Dokl. Akad. Nauk SSSR, 174:19-20, 1967.

[8] Deirdre Haskell, Ehud Hrushovski, and Dugald Macpherson. Definable sets in algebraically closed valued fields: elimination of imaginaries. J. Reine Angew. Math., 597:175-236, 2006.

[9] Deirdre Haskell, Ehud Hrushovski, and Dugald Macpherson. Stable domination and independence in algebraically closed valued fields, volume 30 of Lecture Notes in Logic. Association for Symbolic Logic, Chicago, IL, 2008.

[10] Daniel Hoffmann. Witt vectors and separably closed fields with higher derivations. arXiv:1510.00218.

[11] Jizhan Hong. Immediate expansions by valuation of fields. PhD thesis, McMaster University, 2013.

[12] Jizhan Hong. Separably closed valued fields: quantifier elimination. J. Symbolic Logic, 81(3):887-900, 2016.

[13] Ehud Hrushovski. The Mordell-Lang conjecture for function fields. J. Amer. Math. Soc., 9(3):667-690, 1996. 
[14] Ehud Hrushovski and François Loeser. Non-archimedean tame topology and stably dominated types, volume 192 of Annals of Mathematics Studies. Princeton University Press, Princeton, NJ, 2016.

[15] Ehud Hrushovski, Ben Martin, and Silvain Rideau. Zeta functions from definable equivalence relations. To appear in J. Eur. Math. Soc. (arXiv:math/0701011v4, with an appendix by Raf Cluckers).

[16] Ehud Hrushovski and Anand Pillay. On NIP and invariant measures. J. Eur. Math. Soc. (JEMS), 13(4):1005-1061, 2011.

[17] Franz-Viktor Kuhlmann. Valuation theory. http://math.usask.ca/ fvk/Fvkbook.htm.

[18] T. Mellor. Imaginaries in real closed valued fields. Ann. Pure Appl. Logic, 139(1-3):230-279, 2006.

[19] James S. Milne. Étale cohomology. Number 33 in Princeton Mathematical Series. Princeton University Press, Princeton, N.J., 1980.

[20] James S. Milne. Lectures on etale cohomology. Course lecture notes, 32013.

[21] Bruno Poizat. Paires de structures stables. J. Symbolic Logic, 48(2):239-249, 1983.

[22] Alexander Prestel and Martin Ziegler. Model-theoretic methods in the theory of topological fields. J. Reine Angew. Math., 299(300):318-341, 1978.

[23] Silvain Rideau. Imaginaries and invariant types in existentially closed valued differential fields. J. Reine Angew. Math., 2016.

[24] Silvain Rideau and Pierre Simon. Definable and invariant types in enrichments of NIP theories. J. Symb. Log., 82(1):317-324, 2017.

[25] Thomas Scanlon. A model complete theory of valued D-fields. J. Symbolic Logic, 65(4):1758$1784,2000$.

[26] S. Shelah. Classification theory and the number of nonisomorphic models, volume 92 of Studies in Logic and the Foundations of Mathematics. North-Holland Publishing Co., Amsterdam, second edition, 1990.

[27] Pierre Simon. A guide to NIP theories, volume 44 of Lecture Notes in Logic. Association for Symbolic Logic, Chicago, IL; Cambridge University Press, Cambridge, 2015.

[28] Lou van den Dries. Dimension of definable sets, algebraic boundedness and Henselian fields. Ann. Pure Appl. Logic, 45(2):189-209, 1989. Stability in model theory, II (Trento, 1987).

[29] Carol Wood. Notes on the stability of separably closed fields. J. Symbolic Logic, 44(3):412416, 1979.

[30] Martin Ziegler. Separably closed fields with Hasse derivations. J. Symbolic Logic, 68(1):311$318,2003$.

Westfälische Wilhelms-Universität MÜnster, Institut für Mathematische Logik und Grundlagenforschung, Einsteinstr. 62, D-48149 Münster, Germany

E-mail address: hils@uni-muenster.de

Department of Mathematics, Ben-Gurion University, Be'er-Sheva, Israel

E-mail address: kamenskm@math.bgu.ac.il

University of California, Berkeley, Mathematics Department, Evans Hall, BerkeLey, CA, 94720-3840, United States of America

E-mail address: silvain.rideau@berkeley.edu 